\title{
The Christoffel-Darboux Kernel
}

\author{
Barry Simon*
}

\begin{abstract}
A review of the uses of the CD kernel in the spectral theory of orthogonal polynomials, concentrating on recent results.
\end{abstract}

\section{Contents}

1. Introduction

2. The ABC Theorem

3. The Christoffel-Darboux Formula

4. Zeros of OPRL: Basics Via CD

5. The CD Kernel and Formula for MOPs

6. Gaussian Quadrature

7. Markov-Stieltjes Inequalities

8. Mixed CD Kernels

9. Variational Principle: Basics

10. The Nevai Class: An Aside

11. Delta Function Limits of Trial Polynomials

12. Regularity: An Aside

13. Weak Limits

14. Variational Principle: Máté-Nevai Upper Bounds

15. Criteria for A.C. Spectrum

16. Variational Principle: Nevai Trial Polynomial

17. Variational Principle: Máté-Nevai-Totik Lower Bound

18. Variational Principle: Polynomial Maps

19. Floquet-Jost Solutions for Periodic Jacobi Matrices 29

20. Lubinsky's Inequality and Bulk Universality 29

21. Derivatives of CD Kernels 30

22. Lubinsky's Second Approach 31

23. Zeros: The Freud-Levin-Lubinsky Argument 34

24. Adding Point Masses 35

References 37

2000 Mathematics Subject Classification. 34L40, 47-02, 42 C05.

Key words and phrases. Orthogonal polynomials, spectral theory.

This work was supported in part by NSF grant DMS-0652919 and U.S.-Israel Binational Science Foundation (BSF) Grant No. 2002068. 


\section{Introduction}

This article reviews a particular tool of the spectral theory of orthogonal polynomials. Let $\mu$ be a measure on $\mathbb{C}$ with finite moments, that is,

$$
\int|z|^{n} d \mu(z)<\infty
$$

for all $n=0,1,2, \ldots$ and which is nontrivial in the sense that it is not supported on a finite set of points. Thus, $\left\{z^{n}\right\}_{n=0}^{\infty}$ are independent in $L^{2}(\mathbb{C}, d \mu)$, so by GramSchmidt, one can define monic orthogonal polynomials, $X_{n}(z ; d \mu)$, and orthonormal polynomials, $x_{n}=X_{n} /\left\|X_{n}\right\|_{L^{2}}$. Thus,

$$
\begin{gathered}
\int \bar{z}^{j} X_{n}(z ; d \mu) d \mu(z)=0 \quad j=0, \ldots, n-1 \\
X_{n}(z)=z^{n}+\text { lower order } \\
\int \overline{x_{n}(z)} x_{m}(z) d \mu=\delta_{n m}
\end{gathered}
$$

We will often be interested in the special cases where $\mu$ is supported on $\mathbb{R}$ (especially with support compact), in which case we use $P_{n}, p_{n}$ rather than $X_{n}, x_{n}$, and where $\mu$ is supported on $\partial \mathbb{D}(\mathbb{D}=\{z|| z \mid<1\})$, in which case we use $\Phi_{n}, \varphi_{n}$. We call these OPRL and OPUC (for "real line" and "unit circle").

OPRL and OPUC are spectral theoretic because there are Jacobi parameters $\left\{a_{n}, b_{n}\right\}_{n=1}^{\infty}$ and Verblunsky coefficients $\left\{\alpha_{n}\right\}_{n=0}^{\infty}$ with recursion relations $\left(p_{-1}=0\right.$; $\left.p_{0}=\Phi_{0}=1\right)$ :

$$
\begin{aligned}
z p_{n}(z) & =a_{n+1} p_{n+1}(z)+b_{n+1} p_{n}(z)+a_{n} p_{n-1}(z) \\
\Phi_{n+1}(z) & =z \Phi_{n}(z)-\bar{\alpha}_{n} \Phi_{n}^{*}(z) \\
\Phi_{n}^{*}(z) & =z^{n} \overline{\Phi_{n}(1 / \bar{z})}
\end{aligned}
$$

We will sometimes need the monic OPRL and normalized OPUC recursion relations:

$$
\begin{aligned}
& z P_{n}(z)=P_{n+1}(z)+b_{n+1} P_{n}(z)+a_{n}^{2} P_{n-1}(z) \\
& z \varphi_{n}(z)=\rho_{n} \varphi_{n+1}(z)+\bar{\alpha}_{n} \varphi_{n}^{*}(z) \\
& \rho_{n} \equiv\left(1-\left|\alpha_{n}\right|^{2}\right)^{1 / 2}
\end{aligned}
$$

Of course, the use of $\rho_{n}$ implies $\left|\alpha_{n}\right|<1$ and all sets of $\left\{\alpha_{n}\right\}_{n=0}^{\infty}$ obeying this occur. Similarly, $b_{n} \in \mathbb{R}, a_{n} \in(0, \infty)$ and all such sets occur. In the OPUC case, $\left\{\alpha_{n}\right\}_{n=0}^{\infty}$ determine $d \mu$, while in the OPRL case, they do if $\sup \left(\left|a_{n}\right|+\left|b_{n}\right|\right)<\infty$, and may or may not in the unbounded case. For basics of OPRL, see [93, 22, 34, 89; and for basics of OPUC, see [93, 37, 34, 80, 81, 79.

We will use $\kappa_{n}$ (or $\kappa_{n}(d \mu)$ ) for the leading coefficient of $x_{n}, p_{n}$, or $\varphi_{n}$, so

$$
\kappa_{n}=\left\|X_{n}\right\|_{L^{2}(d \mu)}^{-1}
$$

The Christoffel-Darboux kernel (named after $[\mathbf{2 3}, \mathbf{2 8}$ ) is defined by

$$
K_{n}(z, \zeta)=\sum_{j=0}^{n} \overline{x_{j}(z)} x_{j}(\zeta)
$$


We sometimes use $K_{n}(z, \zeta ; \mu)$ if we need to make the measure explicit. Note that if $c>0$,

$$
K_{n}(z, \zeta ; c \mu)=c^{-1} K_{n}(z, \zeta ; \mu)
$$

since $x_{n}(z ; c d \mu)=c^{-1 / 2} x_{n}(z ; d \mu)$.

By the Schwarz inequality, we have

$$
\left|K_{n}(z, \zeta)\right|^{2} \leq K_{n}(z, z) K_{n}(\zeta, \zeta)
$$

There are three variations of convention. Some only sum to $n-1$; this is the more common convention but (1.12) is used by Szegö [93, Atkinson [5], and in 80, 81. As we will note shortly, it would be more natural to put the complex conjugate on $x_{n}(\zeta)$, not $x_{n}(z)$ - and a very few authors do that. For OPRL with $z, \zeta$ real, the complex conjugate is irrelevant-and some authors leave it off even for complex $z$ and $\zeta$.

As a tool in spectral analysis, convergence of OP expansions, and other aspects of analysis, the use of the CD kernel has been especially exploited by Freud and Nevai, and summarized in Nevai's paper on the subject [68. A series of recent papers by Lubinsky (of which [60, 61] are most spectacular) has caused heightened interest in the subject and motivated me to write this comprehensive review.

Without realizing they were dealing with OPRL CD kernels, these objects have been used extensively in the spectral theory community, especially the diagonal kernel

$$
K_{n}(x, x)=\sum_{j=0}^{n}\left|p_{j}(x)\right|^{2}
$$

Continuum analogs of the ratios of this object for the first and second kind polynomials appeared in the work of Gilbert-Pearson $\mathbf{3 8}$ and the discrete analog in Khan-Pearson 49 and then in Jitomirskaya-Last 45. Last-Simon [55] studied $\frac{1}{n} K_{n}(x, x)$ as $n \rightarrow \infty$. Variation of parameters played a role in all these works and it exploits what is essentially mixed CD kernels (see Section 8).

One of our goals here is to emphasize the operator theoretic point of view, which is often underemphasized in the OP literature. In particular, in describing $\mu$, we think of the operator $M_{z}$ on $L^{2}(\mathbb{C}, d \mu)$ of multiplication by $z$ :

$$
\left(M_{z} f\right)(z)=z f(z)
$$

If $\operatorname{supp}(d \mu)$ is compact, $M_{z}$ is a bounded operator defined on all of $L^{2}(\mathbb{C}, d \mu)$. If it is not compact, there are issues of domain, essential selfadjointness, etc. that will not concern us here, except to note that in the OPRL case, they are connected to uniqueness of the solution of the moment problem (see 77]). With this in mind, we use $\sigma(d \mu)$ for the spectrum of $M_{z}$, that is, the support of $d \mu$, and $\sigma_{\text {ess }}(d \mu)$ for the essential spectrum. When dealing with OPRL of compact support (where $M_{z}$ is bounded selfadjoint) or OPUC (where $M_{z}$ is unitary), we will sometimes use $\sigma_{\mathrm{ac}}(d \mu), \sigma_{\mathrm{sc}}(d \mu), \sigma_{\mathrm{pp}}(d \mu)$ for the spectral theory components. (We will discuss $\sigma_{\text {ess }}(d \mu)$ only in the OPUC/OPRL case where it is unambiguous, but for general operators, there are multiple definitions; see [31.)

The basis of operator theoretic approaches to the study of the CD kernel depends on its interpretation as the integral kernel of a projection. In $L^{2}(\mathbb{C}, d \mu)$, the set of polynomials of degree at most $n$ is an $n+1$-dimensional space. We will use 
$\pi_{n}$ for the operator of orthogonal projection onto this space. Note that

$$
\left(\pi_{n} f\right)(\zeta)=\int K_{n}(z, \zeta) f(z) d \mu(z)
$$

The order of $z$ and $\zeta$ is the opposite of the usual for integral kernels and why we mentioned that putting complex conjugation on $x_{n}(\zeta)$ might be more natural in (1.12).

In particular,

$$
\operatorname{deg}(f) \leq n \Rightarrow f(\zeta)=\int K_{n}(z, \zeta) f(z) d \mu(z)
$$

In particular, since $K_{n}$ is a polynomial in $\zeta$ of degree $n$, we have

$$
K_{n}(z, w)=\int K_{n}(z, \zeta) K_{n}(\zeta, w) d \mu(\zeta)
$$

often called the reproducing property.

One major theme here is the frequent use of operator theory, for example, proving the CD formula as a statement about operator commutators. Another theme, motivated by Lubinsky [60, 61], is the study of asymptotics of $\frac{1}{n} K_{n}(x, y)$ on diagonal $(x=y)$ and slightly off diagonal $\left((x-y)=O\left(\frac{1}{n}\right)\right)$.

Sections 2, 3, and 6 discuss very basic formulae, and Sections 4 and 7 simple applications. Sections [5 and 8 discuss extensions of the context of CD kernels. Section 9 starts a long riff on the use of the Christoffel variational principle which runs through Section 23. Section 24 is a final simple application.

Vladimir Maz'ya has been an important figure in the spectral analysis of partial differential operators. While difference equations are somewhat further from his opus, they are related. It is a pleasure to dedicate this article with best wishes on his 70 th birthday.

I would like to thank J. Christiansen for producing Figure 1 (in Section 7) in Maple, and C. Berg, F. Gesztesy, L. Golinskii, D. Lubinsky, F. Marcellán, E. Saff, and V. Totik for useful discussions.

\section{The ABC Theorem}

We begin with a result that is an aside which we include because it deserves to be better known. It was rediscovered and popularized by Berg [11, who found it earliest in a 1939 paper of Collar 24, who attributes it to his teacher, Aitken-so we dub it the ABC theorem. Given that it is essentially a result about GramSchmidt, as we shall see, it is likely it really goes back to the nineteenth century. For applications of this theorem, see $[\mathbf{1 3}, 47$.

$K_{n}$ is a polynomial of degree $n$ in $\bar{z}$ and $\zeta$, so we can define an $(n+1) \times(n+1)$ square matrix, $k^{(n)}$, with entries $k_{j m}^{(n)}, 0 \leq j, m \leq n$, by

$$
K_{n}(z, \zeta)=\sum_{j, m=0}^{n} k_{j m}^{(n)} \bar{z}^{m} \zeta^{j}
$$

One also has the moment matrix

$$
m_{j k}^{(n)}=\left\langle z^{j}, z^{k}\right\rangle=\int \bar{z}^{j} z^{k} d \mu(z)
$$


$0 \leq j, k \leq n$. For OPRL, this is a function of $j+k$, so $m^{(n)}$ is a Hankel matrix. For OPUC, this is a function of $j-k$, so $m^{(n)}$ is a Toeplitz matrix.

Theorem 2.1 (ABC Theorem).

$$
\left(m^{(n)}\right)^{-1}=k^{(n)}
$$

Proof. By (1.18) for $\ell=0, \ldots, n$,

$$
\int K_{n}(z, \zeta) z^{\ell} d \mu(z)=\zeta^{\ell}
$$

Plugging (2.1) in for $K$, using (2.2) to do the integrals leads to

$$
\sum_{j, q=0}^{n} k_{j q}^{(n)} m_{q \ell}^{(n)} \zeta^{j}=\zeta^{\ell}
$$

which says that

$$
\sum_{q} k_{j q}^{(n)} m_{q \ell}^{(n)}=\delta_{j \ell}
$$

which is (2.3).

Here is a second way to see this result in a more general context: Write

$$
x_{j}(z)=\sum_{k=0}^{j} a_{j k} z^{k}
$$

so we can define an $(n+1) \times(n+1)$ triangular matrix $a^{(n)}$ by

$$
a_{j k}^{(n)}=a_{j k}
$$

Then (the Cholesky factorization of $k$ )

$$
k^{(n)}=a^{(n)}\left(a^{(n)}\right)^{*}
$$

with ${ }^{*}$ Hermitean adjoint. The condition

$$
\left\langle x_{j}, x_{\ell}\right\rangle=\delta_{j \ell}
$$

says that

$$
\left(a^{(n)}\right)^{*} m^{(n)}\left(a^{(n)}\right)=\mathbf{1}
$$

the identity matrix. Multiplying by $\left(a^{(n)}\right)^{*}$ on the right and $\left[\left(a^{(n)}\right)^{*}\right]^{-1}$ on the left yields (2.3). This has a clear extension to a general Gram-Schmidt setting.

\section{The Christoffel-Darboux Formula}

The Christoffel-Darboux formula for OPRL says that

$$
K_{n}(z, \zeta)=a_{n+1}\left(\frac{\overline{p_{n+1}(z)} p_{n}(\zeta)-\overline{p_{n}(z)} p_{n+1}(\zeta)}{\bar{z}-\zeta}\right)
$$

and for OPUC that

$$
K_{n}(z, \zeta)=\frac{\overline{\varphi_{n+1}^{*}(z)} \varphi_{n+1}^{*}(\zeta)-\overline{\varphi_{n+1}(z)} \varphi_{n+1}(\zeta)}{1-\bar{z} \zeta}
$$

The conventional wisdom is that there is no CD formula for general OPs, but we will see, in a sense, that is only half true. The usual proofs are inductive. Our proofs here will be direct operator theoretic calculations. 
We focus first on (3.1). From the operator point of view, the key is to note that, by (1.17),

$$
\left\langle g,\left[M_{z}, \pi_{n}\right] f\right\rangle=\int \overline{g(\zeta)}(\bar{\zeta}-z) K_{n}(z, \zeta) f(z) d \mu(\zeta) d \mu(z)
$$

where $[A, B]=A B-B A$. For OPRL, in (3.3), $\zeta$ and $z$ are real, so (3.1) for $z, \zeta \in \sigma(d \mu)$ is equivalent to

$$
\left[M_{z}, \pi_{n}\right]=a_{n+1}\left[\left\langle p_{n}, \cdot\right\rangle p_{n+1}-\left\langle p_{n+1}, \cdot\right\rangle p_{n}\right]
$$

While (3.4) only proves (3.1) for such $\bar{z}, \zeta$ by the fact that both sides are polynomials in $z$ and $\zeta$, it is actually equivalent. Here is the general result:

Theorem 3.1 (General Half CD Formula). Let $\mu$ be a measure on $\mathbb{C}$ with finite moments. Then:

$$
\begin{gathered}
\left(1-\pi_{n}\right)\left[M_{z}, \pi_{n}\right]\left(1-\pi_{n}\right)=0 \\
\pi_{n}\left[M_{z}, \pi_{n}\right] \pi_{n}=0 \\
\left(1-\pi_{n}\right)\left[M_{z}, \pi_{n}\right] \pi_{n}=\frac{\left\|X_{n+1}\right\|}{\left\|X_{n}\right\|}\left\langle x_{n}, \cdot\right\rangle x_{n+1}
\end{gathered}
$$

REMARK. If $\mu$ has compact support, these are formulae involving bounded operators on $L^{2}(\mathbb{C}, d \mu)$. If not, regard $\pi_{n}$ and $M_{z}$ as maps of polynomials to polynomials.

Proof. (3.5) follows from expanding $\left[M_{z}, \pi_{n}\right]$ and using

$$
\pi_{n}\left(1-\pi_{n}\right)=\left(1-\pi_{n}\right) \pi_{n}=0
$$

If we note that $\left[M_{z}, \pi_{n}\right]=-\left[M_{z},\left(1-\pi_{n}\right)\right]$, (3.6) similarly follows from (3.8). By (3.8) again,

$$
\left(1-\pi_{n}\right)\left[M_{z}, \pi_{n}\right] \pi_{n}=\left(1-\pi_{n}\right) M_{z} \pi_{n}
$$

On $\operatorname{ran}\left(\pi_{n-1}\right), \pi_{n}$ is the identity, and multiplication by $z$ leaves one in $\pi_{n}$, that is,

$$
\left(1-\pi_{n}\right) M_{z} \pi_{n}\left\lceil\operatorname{ran}\left(\pi_{n-1}\right)=0\right.
$$

On the other hand, for the monic OPs,

$$
\left(1-\pi_{n}\right) M_{z} \pi_{n} X_{n}=X_{n+1}
$$

since $M_{z} \pi_{n} X_{n}=z^{n+1}+$ lower order and $\left(1-\pi_{n}\right)$ takes any such polynomial to $X_{n+1}$. Since

$$
\frac{\left\|X_{n+1}\right\|}{\left\|X_{n}\right\|}\left\langle x_{n}, X_{n}\right\rangle x_{n+1}=X_{n+1}
$$

we see (3.4) holds on $\operatorname{ran}\left(1-\pi_{n}\right)+\operatorname{ran}\left(\pi_{n-1}\right)+\left[X_{n}\right]$, and so on all of $L^{2}$.

From this point of view, we can understand what is missing for a CD formula for general OP. The missing piece is

$$
\pi_{n}\left[M_{z}, \pi_{n}\right]\left(1-\pi_{n}\right)=\left(\left(1-\pi_{n}\right) M_{z}^{*} \pi_{n}\right)^{*}
$$

The operator on the left of (3.7) is proven to be rank one, but $\left(1-\pi_{n}\right) M_{z}^{*} \pi_{n}$ is, in general, rank $n$. For $\varphi \in \operatorname{ker}\left[\left(1-\pi_{n}\right) M_{z}^{*} \pi_{n}\right] \cap \operatorname{ran}\left(\pi_{n}\right)$ means that $\varphi$ is a polynomial of degree $n$ and so is $\bar{z} \varphi$, at least for a.e. $z$ with respect to $\mu$. Two cases where many $\bar{z} \varphi$ are polynomials of degree $n$-indeed, so many that $\left(1-\pi_{n}\right) M_{z}^{*} \pi_{n}$ is also rank one - are for OPRL where $\bar{z} \varphi=z \varphi$ (a.e. $z \in \sigma(d \mu)$ ) and OPUC where $\bar{z} \varphi=z^{-1} \varphi$ 
(a.e. $z \in \sigma(d \mu)$ ). In the first case, $\bar{z} \varphi \in \operatorname{ran}\left(\pi_{n}\right)$ if $\operatorname{deg}(\varphi) \leq n-1$, and in the second case, if $\varphi(0)=0$.

Thus, only for these two cases do we expect a simple formula for $\left[M_{z}, \pi\right]$.

Theorem 3.2 (CD Formula for OPRL). For OPRL, we have

$$
\left[M_{z}, \pi_{n}\right]=a_{n+1}\left[\left\langle p_{n}, \cdot\right\rangle p_{n+1}-\left\langle p_{n+1}, \cdot\right\rangle p_{n}\right]
$$

and (3.1) holds for $\bar{z} \neq \zeta$.

Proof. Inductively, one has that $p_{n}(x)=\left(a_{1} \ldots a_{n}\right)^{-1} x^{n}+\ldots$, so

$$
\left\|P_{n}\right\|=a_{1} \ldots a_{n} \mu(\mathbb{R})^{1 / 2}
$$

and thus,

$$
\frac{\left\|P_{n+1}\right\|}{\left\|P_{n}\right\|}=a_{n+1}
$$

Moreover, since $M_{z}^{*}=M_{z}$ for OPRL and $[A, B]^{*}=-\left[A^{*}, B^{*}\right]$, we get from (3.12) that

$$
\pi_{n}\left[M_{z}, \pi_{n}\right]\left(1-\pi_{n}\right)=-a_{n+1}\left\langle p_{n+1}, \cdot\right\rangle p_{n}
$$

(3.5)-(3.7), (3.14), and (3.16) imply (3.13) which, as noted, implies (3.1).

For OPUC, the natural object is (note $M_{z} M_{z}^{*}=M_{z}^{*} M_{z}=1$ )

$$
B_{n}=\pi_{n}-M_{z} \pi_{n} M_{z}^{*}=-\left[M_{z}, \pi_{n}\right] M_{z}^{*}
$$

Theorem 3.3 (CD Formula for OPUC). For OPUC, we have

$$
\pi_{n}-M_{z} \pi_{n} M_{z}^{*}=\left\langle\varphi_{n+1}^{*}, \cdot\right\rangle \varphi_{n+1}^{*}-\left\langle\varphi_{n+1}, \cdot\right\rangle \varphi_{n+1}
$$

and (3.2) holds.

Proof. $B_{n}$ is selfadjoint so $\operatorname{ran}\left(B_{n}\right)=\operatorname{ker}\left(B_{n}\right)^{\perp}$. Clearly, $\operatorname{ran}\left(B_{n}\right) \subset$ $\operatorname{ran}\left(\pi_{n}\right)+M_{z}\left[\operatorname{ran}\left(\pi_{n}\right)\right]=\operatorname{ran}\left(\pi_{n+1}\right)$ and $B_{n} z^{\ell}=0$ for $\ell=1, \ldots, n$, so $\operatorname{ran}\left(B_{n}\right)=$ $\left\{z, z^{2}, \ldots, z^{n}\right\}^{\perp} \cap \operatorname{ran}\left(\pi_{n+1}\right)$ is spanned by $\varphi_{n+1}$ and $\varphi_{n+1}^{*}$. Thus, both $B_{n}$ and the right side of (3.18) are rank two selfadjoint operators with the same range and both have trace 0 . Thus, it suffices to find a single vector $\eta$ in the span of $\varphi_{n+1}$ and $\varphi_{n+1}^{*}$ with $B_{n} \eta=($ RHS of (3.18) $) \eta$, since a rank at most one selfadjoint operator with zero trace is zero!

We will take $\eta=z \varphi_{n}$, which lies in the span since, by (1.9) and its *,

$$
\rho_{n} \varphi_{n+1}=z \varphi_{n}-\bar{\alpha}_{n} \varphi_{n}^{*} \quad \rho_{n} \varphi_{n+1}^{*}=\varphi_{n}^{*}-\alpha_{n} z \varphi_{n}
$$

By (3.16), (3.17), and

$$
\left\|\Phi_{n}\right\|=\rho_{0} \ldots \rho_{n-1} \mu(\partial \mathbb{D})
$$

we have that

$$
\begin{aligned}
B_{n}\left(z \varphi_{n}\right) & =\left[\pi_{n}, M_{z}\right] \varphi_{n} \\
& =-\left(1-\pi_{n}\right) M_{z} \pi_{n} \varphi_{n} \\
& =-\rho_{n} \varphi_{n+1}
\end{aligned}
$$

On the other hand, $\varphi_{n+1}^{*} \perp\left\{z, \ldots, z^{n+1}\right\}$, so

$$
\left\langle\varphi_{n+1}^{*}, z \varphi_{n}\right\rangle=0
$$

and, by (3.19),

$$
\left\langle\varphi_{n+1}, z \varphi_{n}\right\rangle=\rho_{n}\left\langle\varphi_{n+1}, \varphi_{n+1}\right\rangle+\bar{\alpha}_{n}\left\langle\varphi_{n+1}, \varphi_{n}^{*}\right\rangle
$$




$$
=\rho_{n}
$$

SO

$$
[\text { LHS of (3.18)] }] z \varphi_{n}=-\rho_{n} \varphi_{n+1}
$$

Note that (3.2) implies

$$
\begin{aligned}
K_{n+1}(z, \zeta)= & K_{n}(z, \zeta)+\overline{\varphi_{n+1}(z)} \varphi_{n+1}(\zeta)\left(\frac{1-\bar{z} \zeta}{1-\bar{z} \zeta}\right) \\
= & \frac{\overline{\varphi_{n+1}^{*}(z)} \varphi_{n+1}^{*}(\zeta)-\bar{z} \zeta \overline{\varphi_{n+1}(z)} \varphi_{n+1}(\zeta)}{1-\bar{z} \zeta}
\end{aligned}
$$

so changing index, we get the "other form" of the CD formula for OPUC,

$$
K_{n}(z, \zeta)=\frac{\overline{\varphi_{n}(z)^{*}} \varphi_{n}^{*}(\zeta)-\overline{z \varphi_{n}(z)} \zeta \varphi_{n}(\zeta)}{1-\bar{z} \varphi}
$$

We also note that Szegó 93 derived the recursion relation from the CD formula, so the lack of a CD formula for general OPs explains the lack of a recursion relation in general.

\section{Zeros of OPRL: Basics Via CD}

In this section, we will use the CD formula to derive the basic facts about the zeros of OPRL. In the vast literature on OPRL, we suspect this is known but we don't know where. We were motivated to look for this by a paper of Wong [105, who derived the basics for zeros of POPUC (paraorthogonal polynomials on the unit circle) using the CD formula (for other approaches to zeros of POPUCs, see [19, [86]). We begin with the CD formula on diagonal:

THEOREM 4.1. For OPRL and $x$ real,

$$
\sum_{j=0}^{n}\left|p_{j}(x)\right|^{2}=a_{n+1}\left[p_{n+1}^{\prime}(x) p_{n}(x)-p_{n}^{\prime}(x) p_{n+1}(x)\right]
$$

Proof. In (3.1) with $z=x, \zeta=y$ both real, subtract $p_{n+1}(y) p_{n}(y)$ from both products on the left and take the limit as $y \rightarrow x$.

Corollary 4.2. If $p_{n}\left(x_{0}\right)=0$ for $x_{0}$ real, then

$$
p_{n+1}\left(x_{0}\right) p_{n}^{\prime}\left(x_{0}\right)<0
$$

Proof. The left-hand side of (4.1) is strictly positive since $p_{0}(x)=1$.

ThEOREM 4.3. All the zeros of $p_{n}(x)$ are real and simple and the zeros of $p_{n+1}$ strictly interlace those of $p_{n}$. That is, between any successive zeros of $p_{n+1}$ lies exactly one zero of $p_{n}$ and it is strictly between, and $p_{n+1}$ has one zero between each successive zero of $p_{n}$ and it has one zero above the top zero of $p_{n}$ and one below the bottom zero of $p_{n}$.

Proof. By (4.2), $p_{n}\left(x_{0}\right)=0 \Rightarrow p_{n}^{\prime}\left(x_{0}\right) \neq 0$, so zeros are simple, which then implies that the sign of $p_{n}^{\prime}$ changes between its successive zeros. By (4.2), the sign of $p_{n+1}$ thus changes between zeros of $p_{n}$, so $p_{n+1}$ has an odd number of zeros between zeros of $p_{n}$. 
$p_{1}$ is a real polynomial, so it has one real zero. For $x$ large, $p_{n}(x)>0$ since the leading coefficient is positive. Thus, $p_{n}^{\prime}\left(x_{0}\right)>0$ at the top zero. From (4.2), $p_{n+1}\left(x_{0}\right)<0$ and thus, since $p_{n+1}(x)>0$ for $x$ large, $p_{n+1}$ has a zero above the top zero of $p_{n}$. Similarly, it has a zero below the bottom zero.

We thus see inductively, starting with $p_{1}$, that $p_{n}$ has $n$ real zeros and they interlace those of $p_{n-1}$.

We note that Ambroladze [3] and then Denisov-Simon [29] used properties of the CD kernel to prove results about zeros (see Wong 105 for the OPUC analog); the latter paper includes:

ThEOREM 4.4. Suppose $a_{\infty}=\sup _{n} a_{n}<\infty$ and $x_{0} \in \mathbb{R}$ has $d=$ $\operatorname{dist}\left(x_{0}, \sigma(d \mu)\right)>0$. Let $\delta=d^{2} /\left(d+\sqrt{2} a_{\infty}\right)$. Then at least one of $p_{n}$ and $p_{n-1}$ has no zeros in $\left(x_{0}-\delta, x_{0}+\delta\right)$.

They also have results about zeros near isolated points of $\sigma(d \mu)$.

\section{The CD Kernel and Formula for MOPs}

Given an $\ell \times \ell$ matrix-valued measure, there is a rich structure of matrix OPs (MOPRL and MOPUC). A huge literature is surveyed and extended in [27. In particular, the CD kernel and CD formula for MORL are discussed in Sections 2.6 and 2.7, and for MOPUC in Section 3.4.

There are two "inner products," maps from $L^{2}$ matrix-valued functions to matrices, $\langle\langle\cdot, \cdot\rangle\rangle_{\mathrm{R}}$ and $\langle\langle\cdot, \cdot\rangle\rangle_{\mathrm{L}}$. The $\mathrm{R}$ for right comes from the form of scalar homogeneity, for example,

$$
\left\langle\langle f, g A\rangle_{\mathrm{R}}=\left\langle\langle f, g\rangle_{\mathrm{R}} A\right.\right.
$$

but $\left\langle\langle f, A g\rangle_{\mathrm{R}}\right.$ is not related to $\left\langle\langle f, g\rangle_{\mathrm{R}}\right.$.

There are two normalized OPs, $p_{j}^{\mathrm{R}}(x)$ and $p_{j}^{\mathrm{L}}(x)$, orthonormal in $\left\langle\langle\cdot, \cdot\rangle_{\mathrm{R}}\right.$ and $\left\langle\langle\cdot, \cdot\rangle_{\mathrm{L}}\right.$, respectively, but a single CD kernel (for $z, w$ real and ${ }^{\dagger}$ is matrix adjoint),

$$
\begin{aligned}
K_{n}(z, w) & =\sum_{k=0}^{n} p_{k}^{\mathrm{R}}(z) p_{k}^{\mathrm{R}}(w)^{\dagger} \\
& =\sum_{k=0}^{n} p_{k}^{\mathrm{L}}(z)^{\dagger} p_{k}^{\mathrm{L}}(w)
\end{aligned}
$$

One has that

$$
\left\langle\left\langle K_{n}(\cdot, z), f(\cdot)\right\rangle\right\rangle_{\mathrm{R}}=\left(\pi_{n} f\right)(z)
$$

where $\pi_{n}$ is the projection in the $\operatorname{Tr}\left(\langle\langle\cdot, \cdot\rangle\rangle_{\mathrm{R}}\right)$ inner product to polynomials of degree $n$.

In [27, the CD formula is proven using Wronskian calculations. We note here that the commutator proof we give in Section 3 extends to this matrix case.

Within the Toeplitz matrix literature community, a result equivalent to the CD formula is called the Gohberg-Semencul formula; see [10, 35, 39, 40, 48, 100, 101. 


\section{Gaussian Quadrature}

Orthogonal polynomials allow one to approximate integrals over a measure $d \mu$ on $\mathbb{R}$ by certain discrete measures. The weights in these discrete measures depend on $K_{n}(x, x)$. Here we present an operator theoretic way of understanding this.

Fix $n$ and, for $b \in \mathbb{R}$, let $J_{n ; F}(b)$ be the $n \times n$ matrix

$$
J_{n ; F}(b)=\left(\begin{array}{ccccc}
b_{1} & a_{1} & 0 & & \\
a_{1} & b_{2} & a_{2} & & \\
0 & a_{2} & b_{3} & & \\
& & & \ddots & \\
& & & & b_{n}+b
\end{array}\right)
$$

(i.e., we truncate the infinite Jacobi matrix and change only the corner matrix element $b_{n}$ to $\left.b_{n}+b\right)$.

Let $\tilde{x}_{j}^{(n)}(b), j=1, \ldots, n$, be the eigenvalues of $J_{n ; F}(b)$ labelled by $\tilde{x}_{1}<\tilde{x}_{2}<$ .... (We shall shortly see these eigenvalues are all simple.) Let $\tilde{\varphi}_{j}^{(n)}$ be the normalized eigenvectors with components $\left[\tilde{\varphi}_{j}^{(n)}(b)\right]_{\ell}, \ell=1, \ldots, n$, and define

$$
\tilde{\lambda}_{j}^{(n)}(b)=\left|\left[\tilde{\varphi}_{j}^{(n)}(b)\right]_{1}\right|^{2}
$$

so that if $e_{1}$ is the vector $(10 \ldots 0)^{t}$, then

$$
\sum_{j=1}^{n} \tilde{\lambda}_{j}^{(n)}(b) \delta_{\tilde{x}_{j}^{(n)}(b)}
$$

is the spectral measure for $J_{n ; F}(b)$ and $e_{1}$, that is,

$$
\left\langle e_{1}, J_{n ; F}(b)^{\ell} e_{1}\right\rangle=\sum_{j=1}^{n} \tilde{\lambda}_{j}^{(n)}(b) \tilde{x}_{j}^{(n)}(b)^{\ell}
$$

for all $\ell$. We are going to begin by proving an intermediate quadrature formula:

THEOREM 6.1. Let $\mu$ be a probability measure. For any $b$ and any $\ell=$ $0,1, \ldots, 2 n-2$,

$$
\int x^{\ell} d \mu=\sum_{j=1}^{n} \tilde{\lambda}_{j}^{(n)}(b) \tilde{x}_{j}^{(n)}(b)^{\ell}
$$

If $b=0$, this holds also for $\ell=2 n-1$.

Proof. For any measure, $\left\{a_{j}, b_{j}\right\}_{j=1}^{n-1}$ determine $\left\{p_{j}\right\}_{j=0}^{n-1}$, and moreover,

$$
\int x\left|p_{n-1}(x)\right|^{2} d \mu=b_{n}
$$

If a measure has finite support with at least $n$ points, one can still define $\left\{p_{j}\right\}_{j=0}^{n-1}$, Jacobi parameters $\left\{a_{j}, b_{j}\right\}_{j=1}^{n-1}$, and $b_{n}$ by (6.6).

$d \mu$ and the measure, call it $d \tilde{\mu}_{1}^{(n)}$, of (6.3) have the same Jacobi parameters $\left\{a_{j}, b_{j}\right\}_{j=1}^{n-1}$, so the same $\left\{p_{j}\right\}_{j=0}^{n-1}$, and thus by

$$
\int x^{k} p_{j}(x) d \mu=0 \quad k=0,1, \ldots, j-1 ; j=1, \ldots, n-1
$$


we inductively get (6.5) for $\ell=0,1,2, \ldots, 2 n-3$. Moreover,

$$
\int p_{n-1}(x)^{2} d \mu=1
$$

determines inductively (6.5) for $\ell=2 n-2$. Finally, if $b=0$, (6.6) yields 6.5 for $\ell=2 n-1$.

As the second step, we want to determine the $\tilde{x}_{j}^{(n)}(b)$ and $\tilde{\lambda}_{j}^{(n)}(b)$.

TheOREM 6.2. Let $K_{n ; F}=\pi_{n-1} M_{z} \pi_{n-1} \uparrow \operatorname{ran}\left(\pi_{n-1}\right)$ for a general finite moment measure, $\mu$, on $\mathbb{C}$. Then

$$
\operatorname{det}_{\operatorname{ran}\left(\pi_{n-1}\right)}\left(z \mathbf{1}-K_{n ; F}\right)=X_{n}(z)
$$

Proof. Suppose $X_{n}(z)$ has a zero of order $\ell$ at $z_{0}$. Let $\varphi=X_{n}(z) /\left(z-z_{0}\right)^{\ell}$. Then, in $\operatorname{ran}\left(\pi_{n}\right)$,

$$
\begin{aligned}
& \left(K_{n ; F}-z_{0}\right)^{j} \varphi \neq 0 \quad j=0,1, \ldots, \ell-1 \\
& \left(K_{n ; F}-z_{0}\right)^{\ell} \varphi=0
\end{aligned}
$$

since $\left(M_{z}-z_{0}\right)^{\ell} \varphi=X_{n}(z)$ and $\pi_{n-1} X_{n}=0$. Thus, $z_{0}$ is an eigenvalue of $K_{n ; F}$ of algebraic multiplicity at least $\ell$. Since $X_{n}(z)$ has $n$ zeros counting multiplicity, this accounts for all the roots, so (6.9) holds because both sides of monic polynomials of degree $n$ with the same roots.

Corollary 6.3. We have for OPRL

$$
\operatorname{det}\left(z-J_{n ; F}(b)\right)=P_{n}(z)-b P_{n-1}(z)
$$

The eigenvalues $\tilde{x}_{j}^{(n)}(b)$ are all simple and obey for $0<b<\infty$ and $j=1, \ldots, n$ (with $\left.\tilde{x}_{n+1}(0)=\infty\right)$,

$$
\tilde{x}_{j}^{(n)}(0)<\tilde{x}_{j}^{(n)}(b)<\tilde{x}_{j+1}^{(n)}(0)
$$

and for $-\infty<b<0$ and $j=1, \ldots, n\left(\right.$ with $\left.\tilde{x}_{n-1}(0)=-\infty\right)$,

$$
\tilde{x}_{j-1}^{(n)}(0)<\tilde{x}_{j}^{(n)}(b)<\tilde{x}_{j}^{(n)}(0)
$$

Proof. (6.12) for $b=0$ is just (6.9). Expanding in minors shows the determinant of $\left(z-J_{n ; F}(b)\right)$ is just the value at $b=0$ minus $b$ times the $(n-1) \times(n-1)$ determinant, proving (6.12) in general.

The inequalities in (6.13) / 6.14) follow either by eigenvalue perturbation theory or by using the arguments in Section 4 .

In fact, our analysis below proves that for $0<b<\infty$,

$$
\tilde{x}_{j}^{(n)}(0)<\tilde{x}_{j}^{(n)}(b)<\tilde{x}_{j}^{(n-1)}(0)
$$

The recursion formula for monic OPs proves that $p_{j}\left(\tilde{x}_{j}(b)\right)$ is the unnormalized eigenvector for $J_{n ; F}(b) . \quad K_{n-1}\left(\tilde{x}_{j}(b), \tilde{x}_{j}(b)\right)^{1 / 2}$ is the normalization constant, so since $p_{0} \equiv 1($ if $\mu(\mathbb{R})=1)$ :

Proposition 6.4. If $\mu(\mathbb{R})=1$, then

$$
\lambda_{j}^{(n)}(b)=\left(K_{n-1}\left(\tilde{x}_{j}^{(n)}(b), \tilde{x}_{j}^{(n)}(b)\right)\right)^{-1}
$$


Now fix $n$ and $x_{0} \in \mathbb{R}$. Define

$$
b\left(x_{0}\right)=\frac{P_{n}\left(x_{0}\right)}{P_{n-1}\left(x_{0}\right)}
$$

with the convention $b=\infty$ if $P_{n-1}\left(x_{0}\right)=0$. Define for $b \neq \infty$,

$$
x_{j}^{(n)}\left(x_{0}\right)=\tilde{x}_{j}^{(n)}\left(b\left(x_{0}\right)\right) \quad j=1, \ldots, n
$$

and if $b\left(x_{0}\right)=\infty$,

$$
x_{j}^{(n)}\left(x_{0}\right)=\tilde{x}_{j}^{(n-1)}(0) \quad j=1, \ldots, n-1
$$

and

$$
\lambda_{j}^{(n)}\left(x_{0}\right)=\left(K_{n-1}\left(x_{j}^{(n)}\left(x_{0}\right), x_{j}^{(n)}\left(x_{0}\right)\right)\right)^{-1}
$$

Then Theorem 6.1 becomes

Theorem 6.5 (Gaussian Quadrature). Fix $n, x_{0}$. Then

$$
\int Q(x) d \mu=\sum_{j=1}^{n} \lambda_{j}^{(n)}\left(x_{0}\right) Q\left(x_{j}^{(n)}\left(x_{0}\right)\right)
$$

for all polynomials $Q$ of degree up to:

(1) $2 n-1$ if $P_{n}\left(x_{0}\right)=0$

(2) $2 n-2$ if $P_{n}\left(x_{0}\right) \neq 0 \neq P_{n-1}\left(x_{0}\right)$

(3) $2 n-3$ if $P_{n-1}\left(x_{0}\right)=0$.

REMarks. 1 . The sum goes to $n-1$ if $P_{n-1}\left(x_{0}\right)=0$.

2. We can define $x_{j}^{(n)}$ to be the solutions of

$$
p_{n-1}\left(x_{0}\right) p_{n}(x)-p_{n}\left(x_{0}\right) p_{n-1}(x)=0
$$

which has degree $n$ if $p_{n-1}\left(x_{0}\right) \neq 0$ and $n-1$ if $p_{n-1}\left(x_{0}\right)=0$.

3. (6.20) makes sense even if $\mu(\mathbb{R}) \neq 1$ and dividing by $\mu(\mathbb{R})$ changes $\int Q(x) d \mu$ and $\lambda_{j}^{(n)}$ by the same amount, so (6.21) holds for all positive $\mu$ (with finite moments), not just the normalized ones.

4. The weights, $\lambda_{j}^{(n)}\left(x_{0}\right)$, in Gaussian quadrature are called Cotes numbers.

\section{Markov-Stieltjes Inequalities}

The ideas of this section go back to Markov 63 and Stieltjes 92 based on conjectures of Chebyshev [21] (see Freud [34).

Lemma 7.1. Fix $x_{1}<\cdots<x_{n}$ in $\mathbb{R}$ distinct and $1 \leq \ell<n$. Then there is a polynomial, $Q$, of degree $2 n-2$ so that

(i)

$$
Q\left(x_{j}\right)= \begin{cases}1 & j=1, \ldots, \ell \\ 0 & 1=\ell+1, \ldots, n\end{cases}
$$

(ii) For all $x \in \mathbb{R}$,

$$
Q(x) \geq \chi_{\left(-\infty, x_{\ell}\right]}(x)
$$

REMARK. Figure 1 has a graph of $Q$ and $\chi_{\left(-\infty, x_{\ell}\right]}$ for $n=5, \ell=3, x_{j}=j-1$. 


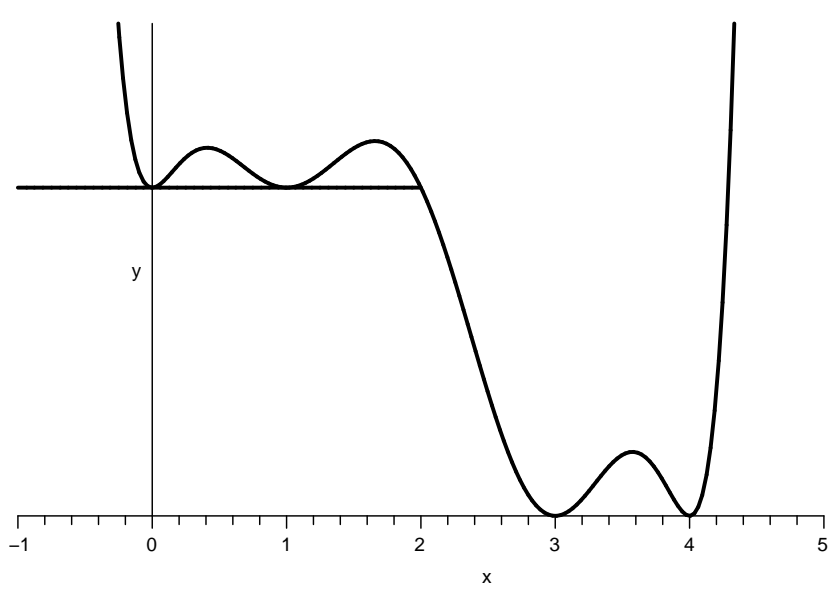

FiguRe 1. An interpolation polynomial

Proof. By standard interpolation theory, there exists a unique polynomial of degree $k$ with $k+1$ conditions of the form

$$
Q\left(y_{j}\right)=Q^{\prime}\left(y_{j}\right)=\cdots=Q^{\left(n_{j}\right)}\left(y_{j}\right)=0
$$

$\sum_{j} n_{j}=k+1$. Let $Q$ be the polynomial of degree $2 n-2$ with the $n$ conditions in (7.1) and the $n-1$ conditions

$$
Q^{\prime}\left(x_{j}\right)=0 \quad j=1, \ldots, \ell-1, \ell+1, \ldots, n
$$

Clearly, $Q^{\prime}$ has at most $2 n-3$ zeros. $n-1$ are given by (7.3) and, by Snell's theorem, each of the $n-2$ intervals $\left(x_{1}, x_{2}\right), \ldots,\left(x_{\ell-1}, x_{\ell}\right),\left(x_{\ell+1}, x_{\ell+2}\right), \ldots,\left(x_{n-1}, x_{n}\right)$ must have a zero. Since $Q^{\prime}$ is nonvanishing on $\left(x_{\ell}, x_{\ell+1}\right)$ and $Q\left(x_{\ell}\right)=1>$ $Q\left(x_{\ell+1}\right)=0, Q^{\prime}(y)<0$ on $\left(x_{\ell}, x_{\ell+1}\right)$. Tracking where $Q^{\prime}$ changes sign, one sees that (7.2) holds.

TheOREm 7.2. Suppose $d \mu$ is a measure on $\mathbb{R}$ with finite moments. Then

$$
\begin{aligned}
& \sum_{\left\{j \mid x_{j}^{(n)}\left(x_{0}\right) \leq x_{0}\right\}} \frac{1}{K_{n-1}\left(x_{j}^{(n)}\left(x_{0}\right), x_{j}^{(n)}\left(x_{0}\right)\right)} \geq \mu\left(\left(-\infty, x_{0}\right]\right) \\
& \quad \geq \mu\left(\left(-\infty, x_{0}\right)\right) \geq \sum_{\left\{j \mid x_{j}^{(n)}\left(x_{0}\right)<x_{0}\right\}} \frac{1}{K_{n-1}\left(x_{j}^{(n)}\left(x_{0}\right), x_{j}^{(n)}\left(x_{0}\right)\right)}
\end{aligned}
$$

Remarks. 1. The two bounds differ by $K_{n-1}\left(x_{0}, x_{0}\right)^{-1}$.

2. These imply

$$
\mu\left(\left\{x_{0}\right\}\right) \leq K_{n-1}\left(x_{0}, x_{0}\right)^{-1}
$$

In fact, one knows (see (9.21) below)

$$
\mu\left(\left\{x_{0}\right\}\right)=\lim _{n \rightarrow \infty} K_{n-1}\left(x_{0}, x_{0}\right)^{-1}
$$

If $\mu\left(\left\{x_{0}\right\}\right)=0$, then the bounds are exact as $n \rightarrow \infty$. 
Proof. Suppose $P_{n-1}\left(x_{0}\right) \neq 0$. Let $\ell$ be such that $x_{\ell}^{(n)}\left(x_{0}\right)=x_{0}$. Let $Q$ be the polynomial of Lemma 7.1 By (7.2),

$$
\mu\left(\left(-\infty, x_{0}\right]\right) \leq \int Q(x) d \mu
$$

and, by (17.1) and Theorem 6.5 the integral is the sum on the left of (7.4).

Clearly, this implies

$$
\mu\left(\left(x_{0}, \infty\right)\right) \geq \sum_{\left\{j \mid x_{j}^{(n)}\left(x_{0}\right)>x_{0}\right\}} \frac{1}{K_{n-1}\left(x_{j}^{(n)}\left(x_{0}\right), x_{j}^{(n)}\left(x_{0}\right)\right)}
$$

which, by $x \rightarrow-x$ symmetry, implies the last inequality in (7.4).

Corollary 7.3. If $\ell \leq k-1$, then

$$
\begin{aligned}
\sum_{j=\ell+1}^{k-1} \frac{1}{K\left(x_{j}^{(n)}\left(x_{0}\right), x_{j}^{(n)}\left(x_{0}\right)\right)} & \leq \mu\left(\left[x_{\ell}^{(n)}\left(x_{0}\right), x_{k}^{(n)}\left(x_{0}\right)\right]\right) \\
& \leq \sum_{j=\ell}^{k} \frac{1}{K\left(x_{j}^{(n)}\left(x_{0}\right), x_{j}^{(n)}\left(x_{0}\right)\right)}
\end{aligned}
$$

Proof. Note if $x_{1}=x_{\ell}^{(n)}\left(x_{0}\right)$ for some $\ell$, then $x_{j}^{(n)}\left(x_{0}\right)=x_{j}^{(n)}\left(x_{1}\right)$, so we get (7.7) by subtracting values of (7.4).

Notice that this corollary gives effective lower bounds only if $k-1 \geq \ell+1$, that is, only on at least three consecutive zeros. The following theorem of Last-Simon [57, based on ideas of Golinskii [41, can be used on successive zeros (see [57] for the proof).

Theorem 7.4. If $E, E^{\prime}$ are distinct zeros of $P_{n}(x), \widetilde{E}=\frac{1}{2}\left(E+E^{\prime}\right)$ and $\delta>$ $\frac{1}{2}\left|E-E^{\prime}\right|$, then

$$
\left|E-E^{\prime}\right| \geq \frac{\delta^{2}-\left(\frac{1}{2}\left|E-E^{\prime}\right|^{2}\right)^{2}}{3 n}\left[\frac{K_{n}(E, E)}{\sup _{|y-\widetilde{E}| \leq \delta} K_{n}(y, y)}\right]^{1 / 2}
$$

\section{Mixed CD Kernels}

Recall that given a measure $\mu$ on $\mathbb{R}$ with finite moments and Jacobi parameters $\left\{a_{n}, b_{n}\right\}_{n=1}^{\infty}$, the second kind polynomials are defined by the recursion relations (1.5) but with initial conditions

$$
q_{0}(x)=0 \quad q_{1}(x)=a_{1}^{-1}
$$

so $q_{n}(x)$ is a polynomial of degree $n-1$. In fact, if $\tilde{\mu}$ is the measure with Jacobi parameters given by

$$
\tilde{a}_{n}=a_{n+1} \quad \tilde{b}_{n}=b_{n+1}
$$

then

$$
q_{n}(x ; d \mu)=a_{1}^{-1} p_{n-1}(x ; d \tilde{\mu})
$$

It is sometimes useful to consider

$$
K_{n}^{(q)}(x, y)=\sum_{j=0}^{n} \overline{q_{j}(x)} q_{j}(y)
$$


and the mixed CD kernel

$$
K_{n}^{(p q)}(x, y)=\sum_{j=0}^{n} \overline{q_{j}(x)} p_{j}(y)
$$

Since (8.2) implies

$$
K_{n}^{(q)}(x, y ; d \mu)=a_{1}^{-2} K_{n-1}(x, y ; d \tilde{\mu})
$$

there is a CD formula for $K^{(q)}$ which follows immediately from the one for $K$. There is also a mixed CD formula for $K_{n}^{(p q)}$.

OPUC also have second kind polynomials, mixed CD kernels, and mixed CD formulae. These are discussed in Section 3.2 of $\mathbf{8 0}$.

Mixed CD kernels will enter in Section 21

\section{Variational Principle: Basics}

If one thing marks the OP approach to the CD kernel that has been missing from the spectral theorists' approach, it is a remarkable variational principle for the diagonal kernel. We begin with:

Lemma 9.1. Fix $\left(\alpha_{1}, \ldots, \alpha_{m}\right) \in \mathbb{C}^{m}$. Then

$$
\min \left(\sum_{j=1}^{m}\left|z_{j}\right|^{2} \mid \sum_{j=1}^{m} \alpha_{j} z_{j}=1\right)=\left(\sum_{j=1}^{m}\left|\alpha_{j}\right|^{2}\right)^{-1}
$$

with the minimizer given uniquely by

$$
z_{j}^{(0)}=\frac{\bar{\alpha}_{j}}{\sum_{j=1}^{m}\left|\alpha_{j}\right|^{2}}
$$

REMARK. One can use Lagrange multipliers to a priori compute $z_{j}^{(0)}$ and prove this result.

PROOF. If

$$
\sum_{j=1}^{m} \alpha_{j} z_{j}=1
$$

then

$$
\sum_{j=1}^{m}\left|z_{j}-z_{j}^{(0)}\right|^{2}=\sum_{j=1}^{m}\left|z_{j}\right|^{2}-\left(\sum_{j=1}^{m}\left|\alpha_{j}\right|^{2}\right)^{-1}
$$

from which the result is obvious.

If $Q$ has $\operatorname{deg}(Q) \leq n$ and $Q_{n}\left(z_{0}\right)=1$, then

$$
Q_{n}(z)=\sum_{j=0}^{n} \alpha_{j} x_{j}(z)
$$

with $x_{j}$ the orthonormal polynomials for a measure $d \mu$, then $\sum \alpha_{j} x_{j}\left(z_{0}\right)=1$ and $\left\|Q_{n}\right\|_{L^{2}(\mathbb{C}, d \mu)}^{2}=\sum_{j=0}^{n}\left|\alpha_{j}\right|^{2}$. Thus the lemma implies:

Theorem 9.2 (Christoffel Variational Principle). Let $\mu$ be a measure on $\mathbb{C}$ with finite moments. Then for $\bar{z}_{0} \in \mathbb{C}$,

$$
\min \left(\int\left|Q_{n}(z)\right|^{2} d \mu \mid Q_{n}\left(z_{0}\right)=1, \operatorname{deg}\left(Q_{n}\right) \leq n\right)=\frac{1}{K_{n}\left(z_{0}, z_{0}\right)}
$$


and the minimizer is given by

$$
Q_{n}\left(z, z_{0}\right)=\frac{K_{n}\left(z_{0}, z\right)}{K_{n}\left(z_{0}, z_{0}\right)}
$$

One immediate useful consequence is:

THEOREM 9.3. If $\mu \leq \nu$, then

$$
K_{n}(z, z ; d \nu) \leq K_{n}(z, z ; d \mu)
$$

For this reason, it is useful to have comparison models:

ExAmple 9.4. Let $d \mu=d \theta / 2 \pi$ for $z=r e^{i \theta}$ and $\zeta=e^{i \varphi}$. We have, since $\varphi_{n}(z)=z^{n}$

$$
K_{n}(z, \zeta)=\frac{1-r^{n+1} e^{i(n+1)(\varphi-\theta)}}{1-r e^{i(\varphi-\theta)}}
$$

If $r<1, K_{n}\left(z, z_{0}\right)$ has a limit as $n \rightarrow \infty$, and for $z=e^{i \varphi}, z_{0}=r e^{i \theta}, r<1$,

$$
\left|Q_{n}\left(z, z_{0}\right)\right|^{2} \frac{d \varphi}{2 \pi} \rightarrow P_{r}(\theta, \varphi) \frac{d \varphi}{2 \pi}
$$

the Poisson kernel,

$$
P_{r}(\theta, \varphi)=\frac{1-r^{2}}{1+r^{2}-2 r \cos (\theta-\varphi)}
$$

For $r=1$, we have

$$
\left|K_{n}\left(e^{i \theta}, e^{i \varphi}\right)\right|^{2}=\frac{\sin ^{2}\left(\frac{n+1}{2}(\theta-\varphi)\right)}{\sin ^{2}(\theta-\varphi)}
$$

the Fejér kernel.

For $r>1$, we use

$$
K_{n}(z, \zeta)=\bar{z}^{n} \zeta^{n} K_{n}\left(\frac{1}{z}, \frac{1}{\zeta}\right)
$$

which implies, for $z=e^{i \varphi}, z_{0}=r e^{i \theta}, r>1$,

$$
\left|Q_{n}\left(z, z_{0}\right)\right|^{2} \frac{d \varphi}{2 \pi} \rightarrow P_{r^{-1}}(\theta, \varphi) \frac{d \varphi}{2 \pi}
$$

EXAMPLE 9.5. Let $d \mu_{0}$ be the measure

$$
d \mu_{0}(x)=\frac{1}{2 \pi} \sqrt{4-x^{2}} \chi_{[-2,2]}(x) d x
$$

on $[-2,2]$. Then $p_{n}$ are the Chebyshev polynomials of the second kind,

$$
p_{n}(2 \cos \theta)=\frac{\sin (n+1) \theta}{\sin \theta}
$$

In particular, if $|x| \leq 2-\delta$,

$$
\left|p_{n}(x+i y)\right| \leq C_{1, \delta} e^{n C_{2, \delta}|y|}
$$

and so

$$
\frac{1}{n}\left|K_{n}(x+i y, x+i y)\right| \leq C_{1, \delta}^{2} e^{2 n C_{2, \delta}|y|}
$$

The following shows the power of the variational principle: 
Theorem 9.6. Let

$$
d \mu=w(x) d x+d \mu_{\mathrm{s}}
$$

Suppose for some $x_{0}, \delta$, we have

$$
w(x) \geq c>0
$$

for $x \in\left[x_{0}-\delta, x_{0}+\delta\right]$. Then for any $\delta^{\prime}<\delta$ and all $x \in\left[x_{0}-\delta^{\prime}, x_{0}+\delta^{\prime}\right]$, we have for all a real,

$$
\frac{1}{n} K_{n}\left(x+\frac{i a}{n}, x+\frac{i a}{n}\right) \leq C_{1} e^{C_{2}|a|}
$$

Proof. We can find a scaled and translated version of the $d \mu_{0}$ of (9.15) with $\mu \geq \mu_{0}$. Now use Theorem 9.3 and (9.18).

The following has many proofs, but it is nice to have a variational one:

TheOREM 9.7. Let $\mu$ be a measure on $\mathbb{R}$ of compact support. For all $x_{0} \in \mathbb{R}$,

$$
\lim _{n \rightarrow \infty} K_{n}\left(x_{0}, x_{0}\right)=\mu\left(\left\{x_{0}\right\}\right)^{-1}
$$

REMARK. If $\mu\left(\left\{x_{0}\right\}\right)=0$, the limit is infinite.

Proof. Clearly, if $Q\left(x_{0}\right)=1, \int\left|Q_{n}(x)\right|^{2} d \mu \geq \mu\left(\left\{x_{0}\right\}\right)$, so

$$
K_{n}\left(x_{0}, x_{0}\right) \leq \mu\left(\left\{x_{0}\right\}\right)^{-1}
$$

On the other hand, pick $A \geq \operatorname{diam}(\sigma(d \mu))$ and let

$$
Q_{2 n}(x)=\left(1-\frac{\left(x-x_{0}\right)^{2}}{A^{2}}\right)^{n}
$$

For any $a$,

$$
\sup _{\substack{\left|x-x_{0}\right| \geq a \\ x \in \sigma(d \mu)}}\left|Q_{2 n}(x)\right| \equiv M_{2 n}(a) \rightarrow 0
$$

so, since $Q_{2 n} \leq 1$ on $\sigma(d \mu)$,

$$
K_{n}\left(x_{0}, x_{0}\right) \geq\left[\mu\left(\left(x_{0}-a, x_{0}+a\right)\right)+M_{2 n}(a)\right]^{-1}
$$

so

$$
\liminf K_{n}\left(x_{0}, x_{0}\right) \geq\left[\mu\left(\left(x_{0}-a, x_{0}+a\right)\right)\right]
$$

for each $a$. Since $\lim _{a \downarrow} \mu\left(\left(x_{0}-a, x_{0}+a\right)\right)=\mu\left(\left\{x_{0}\right\}\right)$, 9.22) and (9.26) imply (9.21).

\section{The Nevai Class: An Aside}

In his monograph, Nevai [67 emphasized the extensive theory that can be developed for OPRL measures whose Jacobi parameters obey

$$
a_{n} \rightarrow a \quad b_{n} \rightarrow b
$$

for some $b$ real and $a>0$. He proved such measures have ratio asymptotics, that is, $P_{n+1}(z) / P_{n}(z)$ has a limit for all $z \in \mathbb{C} \backslash \mathbb{R}$, and Simon [78] proved a converse: Ratio asymptotics at one point of $\mathbb{C}_{+}$implies there are $a, b$, with (10.1). The essential spectrum for such a measure is $[b-2 a, b+2 a]$, so the Nevai class is naturally associated with a single interval $\mathfrak{e} \subset \mathbb{R}$.

The question of what is the proper analog of the Nevai class for a set $\mathfrak{e}$ of the form

$$
\mathfrak{e}=\left[\alpha_{1}, \beta_{1}\right] \cup\left[\alpha_{2}, \beta_{2}\right] \cup \ldots\left[\alpha_{\ell+1}, \beta_{\ell+1}\right]
$$


with

$$
\alpha_{1}<\beta_{1}<\cdots<\alpha_{\ell+1}<\beta_{\ell+1}
$$

has been answered recently and is relevant below.

The key was the realization of López [8, 9] that the proper analog of an arc of a circle was $\left|\alpha_{n}\right| \rightarrow a$ and $\bar{\alpha}_{n+1} \alpha_{n} \rightarrow a^{2}$ for some $a>0$. This is not that $\alpha_{n}$ approaches a fixed sequence but rather that for each $k$,

$$
\min _{e^{i \theta} \in \partial \mathbb{D}} \sum_{j=n}^{n+k}\left|\alpha_{j}-a e^{i \theta}\right| \rightarrow 0
$$

as $n \rightarrow \infty$. Thus, $\alpha_{j}$ approaches a set of Verblunsky coefficients rather than a fixed one.

For any finite gap set $\mathfrak{e}$ of the form $(10.2) /(10.3)$, there is a natural torus, $\mathcal{J}_{\mathfrak{e}}$, of almost periodic Jacobi matrics with $\sigma_{\text {ess }}(J)=\mathfrak{e}$ for all $J \in \mathcal{J}_{\mathfrak{e}}$. This can be described in terms of minimal Herglotz functions [90, 89] or reflectionless two-sided Jacobi matrices [75. All $J \in \mathcal{J}_{\mathfrak{e}}$ are periodic if and only if each $\left[\alpha_{j}, \beta_{j}\right]$ has rational harmonic measure. In this case, we say $\mathfrak{e}$ is periodic.

DEFINITION.

$$
\begin{gathered}
d_{m}\left(\left\{a_{n}, b_{n}\right\}_{n=1}^{\infty},\left\{\tilde{a}_{n}, \tilde{b}_{n}\right\}_{n=1}^{\infty}\right)=\sum_{j=0}^{\infty} e^{-j}\left(\left|a_{m+j}-\tilde{a}_{m+j}\right|+\left|b_{m+j}-\tilde{b}_{m+j}\right|\right) \\
d_{m}\left(\left\{a_{n}, b_{n}\right\}, \mathcal{J}_{\mathfrak{e}}\right)=\min _{J \in \mathcal{J}_{\mathfrak{e}}} d_{m}\left(\left\{a_{n}, b_{n}\right\}, J\right)
\end{gathered}
$$

Definition. The Nevai class for $\mathfrak{e}, N(\mathfrak{e})$, is the set of all Jacobi matrices, $J$, with

$$
d_{m}\left(J, \mathcal{J}_{\mathfrak{e}}\right) \rightarrow 0
$$

as $m \rightarrow \infty$.

This definition is implicit in Simon 81; the metric $d_{m}$ is from 26. Notice that in case of a single gap $\mathfrak{e}$ in $\partial \mathbb{D}$, the isospectral torus is the set of $\left\{\alpha_{n}\right\}_{n=0}^{\infty}$ with $\alpha_{n}=a e^{i \theta}$ for all $n$ where $a$ is $\mathfrak{e}$ dependent and fixed and $\theta$ is arbitrary. The above definition is the López class.

That this is the "right" definition is seen by the following pair of theorems:

Theorem 10.1 (Last-Simon [56]). If $J \in N(\mathfrak{e})$, then

$$
\sigma_{\text {ess }}(J)=\mathfrak{e}
$$

Theorem 10.2 ([26 for periodic e's; $\mathbf{7 5}$ in general). If

$$
\sigma_{\text {ess }}(J)=\sigma_{\text {ac }}(J)=\mathfrak{e}
$$

then $J \in N(\mathfrak{e})$.

\section{Delta Function Limits of Trial Polynomials}

Intuitively, the minimizer, $Q_{n}\left(x, x_{0}\right)$, in the Christoffel variational principle must be 1 at $z_{0}$ and should try to be small on the rest of $\sigma(d \mu)$. As the degree gets larger and larger, one expects it can do this better and better. So one might guess that for every $\delta>0$,

$$
\sup _{\substack{\left|x-x_{0}\right|>\delta \\ x \in \sigma(d \mu)}}\left|Q_{n}\left(x, x_{0}\right)\right| \rightarrow 0
$$


While this happens in many cases, it is too much to hope for. If $x_{1} \in \sigma(d \mu)$ but $\mu$ has very small weight near $x_{1}$, then it may be a better strategy for $Q_{n}$ not to be small very near $x_{1}$. Indeed, we will see (Example 11.3) that the sup in (11.1) can go to infinity. What is more likely is to expect that $\left|Q_{n}\left(x, x_{0}\right)\right|^{2} d \mu$ will be concentrated near $x_{0}$. We normalize this to define

$$
d \eta_{n}^{\left(x_{0}\right)}(x)=\frac{\left|Q_{n}\left(x, x_{0}\right)\right|^{2} d \mu(x)}{\int\left|Q_{n}\left(x, x_{0}\right)\right|^{2} d \mu(x)}
$$

so, by (9.6) / (9.7), in the OPRL case,

$$
d \eta_{n}^{\left(x_{0}\right)}(x)=\frac{\left|K_{n}\left(x, x_{0}\right)\right|^{2}}{K_{n}\left(x, x_{0}\right)} d \mu(x)
$$

We say $\mu$ obeys the Nevai $\delta$-convergence criterion if and only if, in the sense of weak (aka vague) convergence of measures,

$$
d \eta_{n}^{\left(x_{0}\right)}(x) \rightarrow \delta_{x_{0}}
$$

the point mass at $x_{0}$. In this section, we will explore when this holds.

Clearly, if $x_{0} \notin \sigma(d \mu)$, (11.4) cannot hold. We saw, for OPUC with $d \mu=d \theta / 2 \pi$ and $z \notin \partial \mathbb{D}$, the limit was a Poisson measure, and similar results should hold for suitable OPRL. But we will see below (Example 11.2) that even on $\sigma(d \mu)$, (11.4) can fail. The major result below is that for Nevai class on $\mathfrak{e}^{\text {int }}$, it does hold. We begin with an equivalent criterion:

Definition. We say Nevai's lemma holds if

$$
\lim _{n \rightarrow \infty} \frac{\left|p_{n}\left(x_{0}\right)\right|^{2}}{K_{n}\left(x_{0}, x_{0}\right)}=0
$$

TheOREM 11.1. If $d \mu$ is a measure on $\mathbb{R}$ with bounded support and

$$
\inf _{n} a_{n}>0
$$

then for any fixed $x_{0} \in \mathbb{R}$,

$$
\text { (11.4) } \Leftrightarrow \text { (11.5) }
$$

Remark. That (11.5) $\Rightarrow(11.4)$ is in Nevai 67]. The equivalence is a result of Breuer-Last-Simon 14.

Proof. Since

$$
\begin{gathered}
1-\frac{K_{n-1}\left(x_{0}, x_{0}\right)}{K_{n}\left(x_{0}, x_{0}\right)}=\frac{\left|p_{n}\left(x_{0}\right)\right|^{2}}{K_{n}\left(x_{0}, x_{0}\right)} \\
\Leftrightarrow \frac{K_{n-1}\left(x_{0}, x_{0}\right)}{K_{n}\left(x_{0}, x_{0}\right)} \rightarrow 1
\end{gathered}
$$

so

$$
\text { (11.5) } \Rightarrow \frac{\left|p_{n+1}\left(x_{0}\right)\right|^{2}}{K_{n}\left(x_{0}, x_{0}\right)}=\frac{\left|p_{n+1}\left(x_{0}\right)\right|^{2}}{K_{n+1}\left(x_{0}, x_{0}\right)} \frac{K_{n+1}\left(x_{0}, x_{0}\right)}{K_{n}\left(x_{0}, x_{0}\right)} \rightarrow 0
$$

We thus conclude

$$
\text { (11.5) } \Leftrightarrow \frac{\left|p_{n}\left(x_{0}\right)\right|^{2}+\left|p_{n+1}\left(x_{0}\right)\right|^{2}}{K_{n}\left(x_{0}, x_{0}\right)} \rightarrow 0
$$

By the CD formula and orthonormality of $p_{j}(x)$,

$$
\int\left|x-x_{0}\right|^{2}\left|K_{n}\left(x, x_{0}\right)\right|^{2} d \mu=a_{n+1}^{2}\left[p_{n}\left(x_{0}\right)^{2}+p_{n+1}\left(x_{0}\right)^{2}\right]
$$


so, by (11.6) and (11.10),

$$
\int\left|x-x_{0}\right|^{2} d \eta_{n}^{\left(x_{0}\right)}(x) \rightarrow 0 \Leftrightarrow \text { (11.5) }
$$

when $a_{n}$ is uniformly bounded above and away from zero. But since $d \eta_{n}$ have support in a fixed interval,

$$
\text { (11.4) } \Leftrightarrow \int\left|x-x_{0}\right|^{2} d \eta_{n}^{\left(x_{0}\right)} \rightarrow 0
$$

EXAMPLE 11.2. Suppose at some point $x_{0}$, we have

$$
\lim _{n \rightarrow \infty}\left(\left|p_{n}\left(x_{0}\right)\right|^{2}+\left|p_{n+1}\left(x_{0}\right)\right|^{2}\right)^{1 / n} \rightarrow A>1
$$

We claim that

$$
\limsup _{n \rightarrow \infty} \frac{\left|p_{n}\left(x_{0}\right)\right|^{2}}{K_{n}\left(x_{0}, x_{0}\right)}>0
$$

for if (11.12) fails, then (11.5) holds and, by (11.7), for any $\varepsilon$, we can find $N_{0}$ so for $n \geq N_{0}$,

$$
K_{n+1}\left(x_{0}, x_{0}\right) \leq(1+\varepsilon) K_{n}\left(x_{0}, x_{0}\right)
$$

so

$$
\lim K_{n}\left(x_{0}, x_{0}\right)^{1 / n} \leq 1
$$

So, by (11.5), (11.11) fails. Thus, (11.11) implies that (11.5) fails, and so (11.4) fails.

REMARK. As the proof shows, rather than a limit in (11.12), we can have a $\lim \inf >1$.

The first example of this type was found by Szwarc 94. He has a $d \mu$ with pure points at $2-n^{-1}$ but not at 2 , and so that the Lyapunov exponent at 2 was positive but 2 was not an eigenvalue, so (11.11) holds. The Anderson model (see 20]) provides a more dramatic example. The spectrum is an interval $[a, b]$ and (11.11) holds for a.e. $x \in[a, b]$. The spectral measure in this case is supported at eigenvalues and at eigenvalues (11.8), and so (11.4) holds. Thus (11.4) holds on a dense set in $[a, b]$ but fails for Lebesgue a.e. $x_{0}$ !

Example 11.3. A Jacobi weight has the form

$$
d \mu(x)=C_{a, b}(1-x)^{a}(1+x)^{b} d x
$$

with $a, b>-1$. In general, one can show 93

$$
p_{n}(1) \sim c n^{a+1 / 2}
$$

so if $x_{0} \in(-1,1)$ where $\left|p_{n}\left(x_{0}\right)\right|^{2}+\left|p_{n-1}\left(x_{0}\right)\right|^{2}$ is bounded above and below, one has

$$
\frac{\left|K_{n}\left(x_{0}, 1\right)\right|}{K_{n}\left(x_{0}, x_{0}\right)} \sim \frac{n^{a+1 / 2}}{n}=n^{a-1 / 2}
$$

so if $a>\frac{1}{2},\left|Q_{n}\left(x_{0}, 1\right)\right| \rightarrow \infty$. Since $d \mu(x)$ is small for $x$ near 1 , one can (and, as we will see, does) have (11.4) even though (11.1) fails.

With various counterexamples in place (and more later!), we turn to the positive results: 
Theorem 11.4 (Nevai 67, Nevai-Totik-Zhang [69]). If $d \mu$ is a measure in the classical Nevai class (i.e., for a single interval, $\mathfrak{e}=[b-2 a, b+2 a]$ ), then (11.5) and so (11.4) holds uniformly on $\mathfrak{e}$.

Theorem 11.5 (Zhang [108, Breuer-Last-Simon [14). Let $\mathfrak{e}$ be a periodic finite gap set and let $\mu$ lie in the Nevai class for $\mathfrak{e}$. Then (11.5) and so (11.4) holds uniformly on $\mathfrak{e}$.

THEOREM 11.6 (Breuer-Last-Simon [14]). Let $\mathfrak{e}$ be a general finite gap set and let $\mu$ lie in the Nevai class for $\mathfrak{e}$. Then (11.5) and so (11.4) holds uniformly on compact subsets of $\mathfrak{e}^{\mathrm{int}}$.

Remarks. 1. Nevai 67] proved (10.4)/(10.5) for the classical Nevai class for every energy in $\mathfrak{e}$ but only uniformly on compacts of $\mathfrak{e}^{\text {int }}$. Uniformity on all of $\mathfrak{e}$ using a beautiful lemma is from $6 \mathbf{6 9}$.

2. Zhang 108 proved Theorem 11.5 for any $\mu$ whose Jacobi parameters approached a fixed periodic Jacobi matrix. Breuer-Last-Simon [14 noted that without change, Zhang's result holds for the Nevai class.

3. It is hoped that the final version of $\mathbf{1 4}$ will prove the result in Theorem 11.6 on all of $\mathfrak{e}$, maybe even uniformly in $\mathfrak{e}$.

EXAMPLE 11.7 ([14]). In the next section, we will discuss regular measures. They have zero Lyapunov exponent on $\sigma_{\text {ess }}(\mu)$, so one might expect Nevai's lemma could hold - and it will in many regular cases. However, 14 prove that if $b_{n} \equiv 0$ and $a_{n}$ is alternately 1 and $\frac{1}{2}$ on successive very long blocks ( 1 on blocks of size $3^{n^{2}}$ and $\frac{1}{2}$ on blocks of size $\left.2^{n^{2}}\right)$, then $d \mu$ is regular for $\sigma(d \mu)=[-2,2]$. But for a.e. $x \in[-2,2] \backslash[-1,1]$, (10.4) and (10.3) fail.

Conjecture 11.8 (14]). The following is extensively discussed in [14]: For general OPRL of compact support and a.e. $x$ with respect to $\mu$, (10.4) and so (10.3) holds.

\section{Regularity: An Aside}

There is another class besides the Nevai class that enters in variational problems because it allows exponential bounds on trial polynomials. It relies on notions from potential theory; see $[\mathbf{4 2}, \mathbf{5 2}, \mathbf{7 3}, \mathbf{1 0 2}$ for the general theory and $[\mathbf{9 1}, \mathbf{8 5}$. for the theory in the context of orthogonal polynomials.

Definition. Let $\mu$ be a measure with compact support and let $\mathfrak{e}=\sigma_{\text {ess }}(\mu)$. We say $\mu$ is regular for $\mathfrak{e}$ if and only if

$$
\lim _{n \rightarrow \infty}\left(a_{1} \ldots a_{n}\right)^{1 / n}=C(\mathfrak{e})
$$

the capacity of $\mathfrak{e}$.

For $\mathfrak{e}=[-1,1], C(\mathfrak{e})=\frac{1}{2}$ and the class of regular measures was singled out initially by Erdős-Turán $\mathbf{3 2}$ and extensively studied by Ullman $\mathbf{1 0 3}$. The general theory was developed by Stahl-Totik 91 .

Recall that any set of positive capacity has an equilibrium measure, $\rho_{\mathfrak{e}}$, and Green's function, $G_{\mathfrak{e}}$, defined by requiring $G_{\mathfrak{e}}$ is harmonic on $\mathbb{C} \backslash \mathfrak{e}, G_{\mathfrak{e}}(z)=\log |z|+$ $O(1)$ near infinity, and for quasi-every $x \in \mathfrak{e}$,

$$
\lim _{z_{n} \rightarrow x} G_{\mathfrak{e}}\left(z_{n}\right)=0
$$


(quasi-every means except for a set of capacity 0). $\mathfrak{e}$ is called regular for the Dirichlet problem if and only if (12.2) holds for every $x \in \mathfrak{e}$. Finite gap sets are regular for the Dirichlet problem.

One major reason regularity will concern us is:

THEOREM 12.1. Let $\mathfrak{e} \subset \mathbb{R}$ be compact and regular for the Dirichlet problem. Let $\mu$ be a measure regular for $\mathfrak{e}$. Then for any $\varepsilon$, there is $\delta>0$ and $C_{\varepsilon}$ so that

$$
\sup _{\operatorname{dist}(z, \mathfrak{e})<\delta}\left|p_{n}(z, d \mu)\right| \leq C_{\varepsilon} e^{\varepsilon|n|}
$$

For proofs, see [91, 85. Since $K_{n}$ has $n+1$ terms, 12.3) implies

$$
\sup _{\substack{\operatorname{dist}(z, \mathfrak{e})<\delta \\ \operatorname{dist}(w, \mathfrak{e})<\delta}}\left|K_{n}(z, w)\right| \leq(n+1) C_{\varepsilon}^{2} e^{2 \varepsilon|n|}
$$

and for the minimum (since $K_{n}\left(z_{0}, z_{0}\right) \geq 1$ ),

$$
\sup _{\substack{\operatorname{dist}(z, \mathfrak{e})<\delta \\ \operatorname{dist}\left(z_{0}, \mathfrak{e}\right)<\delta}}\left|Q_{n}\left(z, z_{0}\right)\right| \leq(n+1) C_{\varepsilon}^{2} e^{2 \varepsilon|n|}
$$

The other reason regularity enters has to do with the density of zeros. If $x_{j}^{(n)}$ are the zeros of $p_{n}(x, d \mu)$, we define the zero counting measure, $d \nu_{n}$, to be the probability measure that gives weight to $n^{-1}$ to each $x_{j}^{(n)}$. For the following, see 91, 85:

THEOREM 12.2. Let $\mathfrak{e} \subset \mathbb{R}$ be compact and let $\mu$ be a regular measure for $\mathfrak{e}$. Then

$$
d \nu_{n} \rightarrow d \rho_{\mathfrak{e}}
$$

the equilibrium measure for $\mathfrak{e}$.

In (12.6), the convergence is weak.

\section{Weak Limits}

A major theme in the remainder of this review is pointwise asymptotics of $\frac{1}{n+1} K_{n}(x, y ; d \mu)$ and its diagonal. Therefore, it is interesting that one can say something about $\frac{1}{n+1} K_{n}(x, x ; d \mu) d \mu(x)$ without pointwise asymptotics. Notice that

$$
d \mu_{n}(x) \equiv \frac{1}{n+1} K_{n}(x, x ; d \mu) d \mu(x)
$$

is a probability measure. Recall the density of zeros, $\nu_{n}$, defined after (12.5).

THEOREM 13.1. Let $\mu$ have compact support. Let $\nu_{n}$ be the density of zeros and $\mu_{n}$ given by (13.1). Then for any $\ell=0,1,2, \ldots$,

$$
\left|\int x^{\ell} d \nu_{n+1}-\int x^{\ell} d \mu_{n}\right| \rightarrow 0
$$

In particular, $d \mu_{n(j)}$ and $d \nu_{n(j)+1}$ have the same weak limits for any subsequence $n(j)$. 
Proof. By Theorem 6.2 the zeros of $P_{n+1}$ are eigenvalues of $\pi_{n} M_{x} \pi_{n}$, so

$$
\int x^{\ell} d \nu_{n+1}=\frac{1}{n+1} \operatorname{Tr}\left(\left(\pi_{n} M_{x} \pi_{n}\right)^{\ell}\right)
$$

On the other hand, since $\left\{p_{j}\right\}_{j=0}^{n}$ is a basis for $\operatorname{ran}\left(\pi_{n}\right)$,

$$
\begin{aligned}
\int x^{\ell} d \mu_{n} & =\frac{1}{n+1} \sum_{j=0}^{n} \int x^{\ell}\left|p_{j}(x)\right|^{2} d \mu(x) \\
& =\frac{1}{n+1} \operatorname{Tr}\left(\pi_{n} M_{x}^{\ell} \pi_{n}\right)
\end{aligned}
$$

It is easy to see that $\left(\pi_{n} M_{x} \pi_{n}\right)^{\ell}-\pi_{n} M_{x}^{\ell} \pi_{n}$ is rank at most $\ell$, so

$$
\text { LHS of }(13.2) \leq \frac{\ell}{n+1}\left\|M_{x}\right\|^{\ell}
$$

goes to 0 as $n \rightarrow \infty$ for $\ell$ fixed.

Remark. This theorem is due to Simon 88 although the basic fact goes back to Avron-Simon [7].

See Simon [88 for an interesting application to comparison theorems for limits of density of states. We immediately have:

Corollary 13.2. Suppose that

$$
d \mu=w(x) d x+d \mu_{\mathrm{s}}
$$

with $d \mu_{\mathrm{s}}$ Lebesgue singular, and on some open interval $I \subset \mathfrak{e}=\sigma_{\mathrm{ess}}(d \mu)$ we have $d \nu_{n} \rightarrow d \nu_{\infty}$ and

$$
d \nu_{\infty} \uparrow I=\nu_{\infty}(x) d x
$$

and suppose that uniformly on $I$,

$$
\lim \frac{1}{n} K_{n}(x, x)=g(x)
$$

and $w(x) \neq 0$ on $I$. Then

$$
g(x)=\frac{\nu_{\infty}(x)}{w(x)}
$$

ProOF. The theorem implies $d \nu_{\infty} \uparrow I=w(x) g(x)$.

Thus, in the regular case, we expect that "usually"

$$
\frac{1}{n} K_{n}(x, x) \rightarrow \frac{\rho_{\mathfrak{e}}(x)}{w(x)}
$$

This is what we explore in much of the rest of this paper.

\section{Variational Principle: Máté-Nevai Upper Bounds}

The Cotes numbers, $\lambda_{n}\left(z_{0}\right)$, are given by (9.6), so upper bounds on $\lambda_{n}\left(z_{0}\right)$ mean lower bounds on diagonal CD kernels and there is a confusion of "upper bounds" and "lower bounds." We will present here some very general estimates that come from the use of trial functions in (9.6) so they are called Máté-Nevai upper bounds (after 65]), although we will write them as lower bounds on $K_{n}$. One advantage is their great generality. 
Definition. Let $d \mu$ be a measure on $\mathbb{R}$ of the form

$$
d \mu=w(x) d x+d \mu_{\mathrm{s}}
$$

where $d \mu_{\mathrm{s}}$ is singular with respect to Lebesgue measure. We call $x_{0}$ a Lebesgue point of $\mu$ if and only if

$$
\begin{gathered}
\frac{n}{2} \mu_{\mathrm{s}}\left(\left[x_{0}-\frac{1}{n}, x_{0}+\frac{1}{n}\right]\right) \rightarrow 0 \\
\frac{n}{2} \int_{x_{0}-\frac{1}{n}}^{x_{0}+\frac{1}{n}}\left|w(x)-w_{0}\left(x_{0}\right)\right| d x \rightarrow 0
\end{gathered}
$$

It is a fundamental fact of harmonic analysis ([76]) that for any $\mu$ Lebesguea.e., $x_{0}$ in $\mathbb{R}$ is a Lebesgue point for $\mu$. Here is the most general version of the $\mathrm{MN}$ upper bound:

TheOREM 14.1. Let $\mathfrak{e} \subset \mathbb{R}$ be an arbitrary compact set which is regular for the Dirichlet problem. Let $I \subset \mathfrak{e}$ be a closed interval. Let $d \mu$ be a measure with compact support in $\mathbb{R}$ with $\sigma_{\mathrm{ess}}(d \mu) \subset \mathfrak{e}$. Then for any Lebesgue point, $x$ in $I$,

$$
\liminf _{n \rightarrow \infty} \frac{1}{n} K_{n}(x, x) \geq \frac{\rho_{\mathfrak{e}}(x)}{w(x)}
$$

where $d \rho_{\mathfrak{e}}\left\lceil I=\rho_{\mathfrak{e}}(x) d x\right.$. If $w$ is continuous on I (including at the endpoints as a function in a neighborhood of $I$ ) and nonvanishing, then (14.4) holds uniformly on I. If $x_{n} \rightarrow x \in I$ and $A=\sup _{n} n\left|x_{n}-x\right|<\infty$ and $x$ is a Lebesgue, then (14.4) holds with $K_{n}(x, x)$ replaced by $K_{n}\left(x_{n}, x_{n}\right)$. If $w$ is continuous and nonvanishing on $I$, then this extended convergence is uniform in $x \in I$ and $x_{n}$ 's with $A \leq A_{0}<\infty$.

Remarks. 1. If $I \subset \mathfrak{e}$ is a nontrivial interval, the measure $d \rho_{\mathfrak{e}} \uparrow I$ is purely absolutely continuous (see, e.g., [85, 89]).

2. For OPUC, this is a result of Máté-Nevai 64. The translation to OPRL on $[-1,1]$ is explicit in Máté-Nevai-Totik [66. The extension to general sets via polynomial mapping and approximation (see Section [18) is due to Totik [96. These papers also require a local Szegő condition, but that is only needed for lower bounds on $\lambda_{n}$ (see Section 17). They also don't state the $x_{n} \rightarrow x_{\infty}$ result, which is a refinement introduced by Lubinsky [60] who implemented it in certain $[-1,1]$ cases.

3. An alternate approach for Totik's polynomial mapping is to use trial functions based on Jost-Floquet solutions for periodic problems; see Section 19 (and also [87, 89]).

One can combine (14.4) with weak convergence and regularity to get

THEOREM 14.2 (Simon $\mathbf{8 8}$ ). Let $\mathfrak{e} \subset \mathbb{R}$ be an arbitrary compact set, regular for the Dirichlet problem. Let $d \mu$ be a measure with compact support in $\mathbb{R}$ with $\sigma_{\mathrm{ess}}(d \mu)=\mathfrak{e}$ and with $d \mu$ regular for $\mathfrak{e}$. Let $I \subset \mathfrak{e}$ be an interval so $w(x)>0$ a.e. on I. Then

$$
\begin{array}{ll}
\text { (i) } & \int_{I}\left|\frac{1}{n} K_{n}(x, x) w(x)-\rho_{\mathfrak{e}}(x)\right| d x \rightarrow 0 \\
\text { (ii) } & \int_{I} \frac{1}{n} K_{n}(x, x) d \mu_{\mathrm{s}}(x) \rightarrow 0
\end{array}
$$


Proof. By Theorems 12.2 and 13.1.

$$
\frac{1}{n} K_{n}(x, x) d \mu \rightarrow d \rho_{\mathfrak{e}}
$$

Let $\nu_{1}$ be a limit point of $\frac{1}{n} K_{n}(x, x) d \mu_{\mathrm{s}}$ and

$$
d \nu_{2}=d \rho_{\mathfrak{e}}-d \nu_{1}
$$

If $f \geq 0$, by Fatou's lemma and (14.4),

$$
\int_{I} f d \nu_{2} \geq \int_{I} \rho_{\mathfrak{e}}(x) f(x) d x
$$

that is, $d \nu_{2}\left\lceil I \geq \rho_{\mathfrak{e}}(x) d x\left\lceil I\right.\right.$. By (14.8), $d \nu_{2}\left\lceil I \leq \rho_{\mathfrak{e}}(x) d x\right.$. It follows $d \nu_{1}\lceil I$ is 0 and $d \nu_{2} \uparrow I=d \rho_{\mathfrak{e}} \uparrow I$.

By compactness, $\frac{1}{n} K_{n}(x, x) d \mu_{\mathrm{s}} \uparrow I \rightarrow 0$ weakly, implying (14.6). By a simple argument 88, weak convergence of $\frac{1}{n} K_{n}(x, x) w(x) d x \rightarrow \rho_{\mathfrak{e}}(x) d x$ and (14.4) imply (14.5).

\section{Criteria for A.C. Spectrum}

Define

$$
N=\left\{x \in \mathbb{R} \mid \liminf \frac{1}{n} K_{n}(x, x)<\infty\right\}
$$

so that

$$
\mathbb{R} \backslash N=\left\{x \in \mathbb{R} \mid \lim \frac{1}{n} K_{n}(x, x)=\infty\right\}
$$

Theorem 14.1 implies

THEOREM 15.1. Let $\mathfrak{e} \subset \mathbb{R}$ be an arbitrary compact set and $d \mu=w(x) d x+d \mu_{\mathrm{s}}$ a measure with $\sigma(\mu)=\mathfrak{e}$. Let $\Sigma_{\mathrm{ac}}=\{x \mid w(x)>0\}$. Then $N \backslash \Sigma_{\mathrm{ac}}$ has Lebesgue measure zero.

Proof. If $x_{0} \in \mathbb{R} \backslash \Sigma_{\mathrm{ac}}$ and is a Lebesgue point of $\mu$, then $w\left(x_{0}\right)=0$ and, by Theorem 14.1 $x_{0} \in \mathbb{R} \backslash N$. Thus,

$$
\left(\mathbb{R} \backslash \Sigma_{\mathrm{ac}}\right) \backslash(\mathbb{R} \backslash N)=N \backslash \Sigma_{\mathrm{ac}}
$$

has Lebesgue measure zero.

Remark. This is a direct but not explicit consequence of the Máté-Nevai ideas 64. Without knowing of this work, Theorem 15.1 was rediscovered with a very different proof by Last-Simon [55].

On the other hand, following Last-Simon [55, we note that Fatou's lemma and

implies

$$
\int \frac{1}{n} K_{n}(x, x) d \mu(x)=1
$$

$$
\int \liminf \frac{1}{n} K_{n}(x, x) d \mu(x) \leq 1
$$

so

THEOREM 15.2 ([55). $\Sigma_{\mathrm{ac}} \backslash N$ has Lebesgue measure zero. 
Thus, up to sets of measure zero, $\Sigma_{\mathrm{ac}}=N$. What is interesting is that this holds, for example, when $\mathfrak{e}$ is a positive measure Cantor set as occurs for the almost Mathieu operator $\left(a_{n} \equiv 1, b_{n}=\lambda \cos (\pi \alpha n+\theta),|\lambda|<2, \lambda \neq 0, \alpha\right.$ irrational). This operator has been heavily studied; see Last [54].

\section{Variational Principle: Nevai Trial Polynomial}

A basic idea is that if $d \mu_{1}$ and $d \mu_{2}$ look alike near $x_{0}$, there is a good chance that $K_{n}\left(x_{0}, x_{0} ; d \mu_{1}\right)$ and $K_{n}\left(x_{0}, x_{0} ; d \mu_{2}\right)$ are similar for $n$ large. The expectation (13.8) says they better have the same support (and be regular for that support), but this is a reasonable guess.

It is natural to try trial polynomials minimizing $\lambda_{n}\left(x_{0}, d \mu_{1}\right)$ in the Christoffel variational principle for $\lambda_{n}\left(x_{0}, d \mu_{2}\right)$, but Example 11.3 shows this will not work in general. If $d \mu_{1}$ has a strong zero near some other $x_{1}$, the trial polynomial for $d \mu_{1}$ may be large near $x_{1}$ and be problematical for $d \mu_{2}$ if it does not have a zero there. Nevai 67. had the idea of using a localizing factor to overcome this.

Suppose $\mathfrak{e} \subset \mathbb{R}$, a compact set which, for now, we suppose contains $\sigma\left(d \mu_{1}\right)$ and $\sigma\left(d \mu_{2}\right)$. Pick $A=\operatorname{diam}(\mathfrak{e})$ and consider (with $[\cdot] \equiv$ integral part)

$$
\left(1-\frac{\left(x-x_{0}\right)^{2}}{A^{2}}\right)^{[\varepsilon n]} \equiv N_{2[\varepsilon n]}(x)
$$

Then for any $\delta$,

$$
\sup _{\substack{\left|x-x_{0}\right|>\delta \\ x \in \mathfrak{e}}} N_{2[\varepsilon n]}(x) \leq e^{-c(\delta, \varepsilon) n}
$$

so if $Q_{n-2[\varepsilon n]}(x)$ is the minimizer for $\mu_{1}$ and $\mathfrak{e}$ is regular for the Dirichlet problem and $\mu_{1}$ is regular for $\mathfrak{e}$, then the Nevai trial function

$$
N_{2[\varepsilon n]}(x) Q_{n-2[\varepsilon n]}(x)
$$

will be exponentially small away from $x_{0}$.

For this to work to compare $\lambda_{n}\left(x_{0}, d \mu_{1}\right)$ and $\lambda\left(x_{0}, d \mu_{2}\right)$, we need two additional properties of $\lambda_{n}\left(x_{0}, d \mu_{1}\right)$ :

(a) $\lambda_{n}\left(x_{0}, d \mu_{1}\right) \geq C_{\varepsilon} e^{-\varepsilon n}$ for each $\varepsilon<0$. This is needed for the exponential contributions away from $x_{0}$ not to matter.

(b)

$$
\lim _{\varepsilon \downarrow 0} \limsup _{n \rightarrow \infty} \frac{\lambda_{n}\left(x_{0}, d \mu_{1}\right)}{\lambda_{n-2[\varepsilon n]}\left(x, d \mu_{1}\right)}=1
$$

so that the change from $Q_{n}$ to $Q_{n-2[\varepsilon n]}$ does not matter.

Notice that both (a) and (b) hold if

$$
\lim _{n \rightarrow \infty} n \lambda_{n}\left(x_{0}, d \mu\right)=c>0
$$

If one only has $\mathfrak{e}=\sigma_{\mathrm{ess}}\left(d \mu_{2}\right)$, one can use explicit zeros in the trial polynomials to mask the eigenvalues outside $\mathfrak{e}$.

For details of using Nevai trial functions, see $\mathbf{8 7}, \mathbf{8 9}$. Below we will just refer to using Nevai trial functions. 


\section{Variational Principle: Máté-Nevai-Totik Lower Bound}

In [66], Máté-Nevai-Totik proved:

TheOREM 17.1. Let $d \mu$ be a measure on $\partial \mathbb{D}$

$$
d \mu=\frac{w(\theta)}{2 \pi} d \theta+d \mu_{\mathrm{s}}
$$

which obeys the Szegö condition

$$
\int \log (w(\theta)) \frac{d \theta}{2 \pi}>-\infty
$$

Then for a.e. $\theta_{\infty} \in \partial \mathbb{D}$,

$$
\liminf n \lambda_{n}\left(\theta_{\infty}\right) \geq w\left(\theta_{\infty}\right)
$$

This remains true if $\lambda_{n}\left(\theta_{\infty}\right)$ is replaced by $\lambda_{n}\left(\theta_{n}\right)$ with $\theta_{n} \rightarrow \theta_{\infty}$ obeying $\sup n \mid \theta_{n}-$ $\theta_{\infty} \mid<\infty$.

Remarks. 1. The proof in $6 \mathbf{6 6}$ is clever but involved (89 has an exposition); it would be good to find a simpler proof. 33 .

2. 66] only has the result $\theta_{n}=\theta_{\infty}$. The general $\theta_{n}$ result is due to Findley

3. The $\theta_{\infty}$ for which this is proven have to be Lebesgue points for $d \mu$ as well as Lebesgue points for $\log (w)$ and for its conjugate function.

4. As usual, if $I$ is an interval with $w$ continuous and nonvanishing, and $\mu_{\mathrm{s}}(I)=$ 0 , (17.3) holds uniformly if $\theta_{\infty} \in I$.

By combining this lower bound with the Máté-Nevai upper bound, we get the result of Máté-Nevai-Totik [66]:

TheOREM 17.2. Under the hypothesis of Theorem [17.1, for a.e. $\theta_{\infty} \in \partial \mathbb{D}$,

$$
\lim _{n \rightarrow \infty} n \lambda_{n}\left(\theta_{\infty}\right)=w\left(\theta_{\infty}\right)
$$

This remains true if $\lambda_{n}\left(\theta_{\infty}\right)$ is replaced by $\lambda_{n}\left(\theta_{n}\right)$ with $\theta_{n} \rightarrow \theta_{\infty}$ obeying $\sup n \mid \theta_{n}-$ $\theta_{\infty} \mid<\infty$. If $I$ is an interval with $w$ continuous on $I$ and $\mu_{\mathrm{s}}(I)=0$, then these results hold uniformly in $I$.

Remark. It is possible (see remarks in Section 4.6 of [68) that (17.4) holds if a Szegő condition is replaced by $w(\theta)>0$ for a.e. $\theta$. Indeed, under that hypothesis, Simon $8 \mathbf{8 8}$ proved that

$$
\int_{0}^{2 \pi}\left|w(\theta)\left(n \lambda_{n}(\theta)\right)^{-1}-1\right| \frac{d \theta}{2 \pi} \rightarrow 0
$$

There have been significant extensions of Theorem 17.2 to OPRL on fairly general sets:

1. 66] used the idea of Nevai trial functions (Section 16) to prove the Szegö condition could be replaced by regularity plus a local Szegö condition.

2. [66] used the Szegö mapping to get a result for $[-1,1]$.

3. Using polynomial mappings (see Section 18) plus approximation, Totik 96 proved a general result (see below); one can replace polynomial mappings by Floquet-Jost solutions (see Section 19) in the case of continuous weights on an interval (see [87).

Here is Totik's general result (extended from $\sigma(d \mu) \subset \mathfrak{e}$ to $\sigma_{\text {ess }}(d \mu) \subset \mathfrak{e}$ ): 
Theorem 17.3 (Totik [96, 99). Let $\mathfrak{e}$ be a compact subset of $\mathbb{R}$. Let $I \subset \mathfrak{e}$ be an interval. Let $d \mu$ have $\sigma_{\mathrm{ess}}(d \mu)=\mathfrak{e}$ be regular for $\mathfrak{e}$ with

$$
\int_{I} \log (w) d x>-\infty
$$

Then for a.e. $x_{\infty} \in I$,

$$
\lim _{n \rightarrow \infty} \frac{1}{n} K_{n}\left(x_{\infty}, x_{\infty}\right)=\frac{\rho_{\mathfrak{e}}\left(x_{\infty}\right)}{w\left(x_{\infty}\right)}
$$

The same limit holds for $\frac{1}{n} K_{n}\left(x_{n}, x_{n}\right)$ if $\sup _{n} n\left|x_{n}-x_{\infty}\right|<\infty$. If $\mu_{\mathrm{s}}(I)=\emptyset$ and $w$ is continuous and nonvanishing on $I$, then those limits are uniform on $x_{\infty} \in I$ and on all $x_{n}$ 's with $\sup _{n} n\left|x_{n}-x_{\infty}\right| \leq A$ (uniform for each fixed $A$ ).

REMARKs. 1. Totik 98 recently proved asymptotic results for suitable CD kernels for OPs which are neither OPUC nor OPRL.

2. The extension to general compact $\mathfrak{e}$ without an assumption of regularity for the Dirichlet problem is in $\mathbf{9 9}$.

\section{Variational Principle: Polynomial Maps}

In passing from $[-1,1]$ to fairly general sets, one uses a three-step process. A finite gap set is an $\mathfrak{e}$ of the form

$$
\mathfrak{e}=\left[\alpha_{1}, \beta_{1}\right] \cup\left[\alpha_{2}, \beta_{2}\right] \cup \cdots \cup\left[\alpha_{\ell+1}, \beta_{\ell+1}\right]
$$

where

$$
\alpha_{1}<\beta_{1}<\alpha_{2}<\beta_{2}<\cdots<\alpha_{\ell+1}<\beta_{\ell+1}
$$

$\mathcal{E}_{f}$ will denote the family of finite gap sets. We write $\mathfrak{e}=\mathfrak{e}_{1} \cup \cdots \cup \mathfrak{e}_{\ell+1}$ in this case with the $\mathfrak{e}_{j}$ closed disjoint intervals. $\mathcal{E}_{p}$ will denote the set of what we called periodic finite gap sets in Section 10 ones where each $\mathfrak{e}_{j}$ has rational harmonic measure. Here are the three steps:

(1) Extend to $\mathfrak{e} \in \mathcal{E}_{p}$ using the methods discussed briefly below.

(2) Prove that given any $\mathfrak{e} \in \mathcal{E}_{f}$, there is $\mathfrak{e}^{(n)} \in \mathcal{E}_{p}$, each with the same number of bands so $\mathfrak{e}_{j} \subset \mathfrak{e}_{j}^{(n)} \subset \mathfrak{e}_{j}^{(n-1)}$ and $\cap_{n} \mathfrak{e}_{j}^{(n)}=\mathfrak{e}_{j}$. This is a result proven independently by Bogatyrëv [12, Peherstorfer [71, and Totik [97; see [89. for a presentation of Totik's method.

(3) Note that for any compact $\mathfrak{e}$, if $\mathfrak{e}^{(m)}=\left\{x \mid \operatorname{dist}(x, \mathfrak{e}) \leq \frac{1}{m}\right\}$, then $\mathfrak{e}^{(m)}$ is a finite gap set and $\mathfrak{e}=\cap_{m} \mathfrak{e}^{(m)}$.

Step (1) is the subtle step in extending theorems: Given the BogatyrëvPeherstorfer-Totik theorem, the extensions are simple approximation.

The key to $\mathfrak{e} \in \mathcal{E}_{p}$ is that there is a polynomial $\tilde{\Delta}: \mathbb{C} \rightarrow \mathbb{C}$, so $\tilde{\Delta}^{-1}([-1,1])=\mathfrak{e}$ and so that $\mathfrak{e}_{j}$ is a finite union of intervals $\tilde{\mathfrak{e}}_{k}$ with disjoint interiors so that $\tilde{\Delta}$ is a bijection from each $\tilde{\mathfrak{e}}_{k}$ to $[-1,1]$. That this could be useful was noted initially by Geronimo-Van Assche [36. Totik showed how to prove Theorem 17.3 for $\mathfrak{e} \in \mathcal{E}_{p}$ from the results for $[-1,1]$ using this polynomial mapping.

For spectral theorists, the polynomial $\tilde{\Delta}=\frac{1}{2} \Delta$ where $\Delta$ is the discriminant for the associated periodic problem (see $[43,53,[104,95,89]$ ). There is a direct construction of $\tilde{\Delta}$ by Aptekarev [4] and Peherstorfer [70, 71, 72]. 


\section{Floquet-Jost Solutions for Periodic Jacobi Matrices}

As we saw in Section 16, models with appropriate behavior are useful input for comparison theorems. Periodic Jacobi matrices have OPs for which one can study the CD kernel and its asymptotics. The two main results concern diagonal and just off-diagonal behavior:

THEOREM 19.1. Let $\mu$ be the spectral measure associated to a periodic Jacobi matrix with essential spectrum, $\mathfrak{e}$, a finite gap set. Let $d \mu=w(x) d x$ on $\mathfrak{e}$ (there can also be up to one eigenvalue in each gap). Then uniformly for $x$ in compact subsets of $\mathfrak{e}^{\text {int }}$,

$$
\frac{1}{n} K_{n}(x, x) \rightarrow \frac{\rho_{\mathfrak{e}}(x)}{w(x)}
$$

and uniformly for such $x$ and $a, b$ in $\mathbb{R}$ with $|a| \leq A,|b| \leq B$,

$$
\frac{K_{n}\left(x+\frac{a}{n}, x+\frac{b}{n}\right)}{K_{n}(x, x)} \rightarrow \frac{\sin \left(\pi \rho_{\mathfrak{e}}(x)(b-a)\right)}{\pi \rho_{\mathfrak{e}}(x)(b-a)}
$$

Remarks. 1. (19.2) is often called bulk universality. On bounded intervals, it goes back to random matrix theory. The best results using Riemann-Hilbert methods for OPs is due to Kuijlaars-Vanlessen 51. A different behavior is expected at the edge of the spectrum - we will not discuss this in detail, but see Lubinsky [62.

2. For $[-1,1]$, Lubinsky [60] used Legendre polynomials as his model. The references for the proofs here are Simon [87, 89.

The key to the proof of Theorem 19.1 is to use Floquet-Jost solutions, that is, solutions of

$$
a_{n} u_{n+1}+b_{n} u_{n}+a_{n-1} u_{n-1}=x u_{n}
$$

for $n \in \mathbb{Z}$ where $\left\{a_{n}, b_{n}\right\}$ are extended periodically to all of $\mathbb{Z}$. These solutions obey

$$
u_{n+p}=e^{i \theta(x)} u_{n}
$$

For $x \in \mathfrak{e}^{\text {int }}, u_{n}$ and $\bar{u}_{n}$ are linearly independent, and so one can write $p_{--1}$ in terms of $u$. and $\bar{u}$.. Using

$$
\rho_{\mathfrak{e}}(x)=\frac{1}{p \pi}\left|\frac{d \theta}{d x}\right|
$$

one can prove (19.1) and (19.2). The details are in [87, $\mathbf{8 9}$.

\section{Lubinsky's Inequality and Bulk Universality}

Lubinsky 60 found a powerful tool for going from diagonal control of the CD kernel to slightly off-diagonal control - a simple inequality.

Theorem 20.1. Let $\mu \leq \mu^{*}$ and let $K_{n}, K_{n}^{*}$ be their $C D$ kernels. Then for any $z, \zeta$,

$$
\left|K_{n}(z, \zeta)-K_{n}^{*}(z, \zeta)\right|^{2} \leq K_{n}(z, z)\left[K_{n}(\zeta, \zeta)-K_{n}^{*}(\zeta, \zeta)\right]
$$

REMARK. Recall (Theorem 9.3) that $K_{n}(\zeta, \zeta) \geq K_{n}^{*}(\zeta, \zeta)$.

Proof. Since $K_{n}-K_{n}^{*}$ is a polynomial $\bar{z}$ of degree $n$ :

$$
K_{n}(z, \zeta)-K_{n}^{*}(z, \zeta)=\int K_{n}(z, w)\left[K_{n}(w, \zeta)-K_{n}^{*}(w, \zeta)\right] d \mu(w)
$$


By the reproducing kernel formula (1.19), we get (20.1) from the Schwarz inequality if we show

$$
\int\left|K_{n}(w, \zeta)-K_{n}^{*}(w, \zeta)\right|^{2} d \mu(w) \leq K_{n}(\zeta, \zeta)-K_{n}^{*}(\zeta, \zeta)
$$

Expanding the square, the $K_{n}^{2}$ term is $K_{n}(\zeta, \zeta)$ by (1.19) and the $K_{n} K_{n}^{*}$ cross term is $-2 K_{n}^{*}(\zeta, \zeta)$ by the reproducing property of $K_{n}$ for $d \mu$ integrals. Thus, (20.3) is equivalent to

$$
\int\left|K_{n}^{*}(w, \zeta)\right|^{2} d \mu(w) \leq K_{n}^{*}(\zeta, \zeta)
$$

This in turn follows from $\mu \leq \mu^{*}$ and (1.19) for $\mu^{*}$ !

This result lets one go from diagonal control on measures to off-diagonal. Given any pair of measures, $\mu$ and $\nu$, there is a unique measure $\mu \vee \nu$ which is their least upper bound (see, e.g., Doob [30]). It is known (see [85]) that if $\mu, \nu$ are regular for the same set, so is $\mu \vee \nu$. (20.1) immediately implies that (go from $\mu$ to $\mu^{*}$ and then $\mu^{*}$ to $\nu$ ):

Corollary 20.2. Let $\mu, \nu$ be two measures and $\mu^{*}=\mu \vee \nu$. Suppose for some $z_{n} \rightarrow z_{\infty}, w_{n} \rightarrow z_{\infty}$, we have for $\eta=\mu, \nu, \mu^{*}$ that

$$
\lim _{n \rightarrow \infty} \frac{K_{n}\left(z_{n}, z_{n} ; \eta\right)}{K_{n}\left(z_{\infty}, z_{\infty} ; \eta\right)}=\lim _{n \rightarrow \infty} \frac{K_{n}\left(w_{n}, w_{n} ; \eta\right)}{K_{n}\left(z_{\infty}, z_{\infty} ; \eta\right)}=1
$$

and that

$$
\lim _{n \rightarrow \infty} \frac{K_{n}\left(z_{\infty}, z_{\infty} ; \mu\right)}{K_{n}\left(z_{\infty}, z_{\infty} ; \mu^{*}\right)}=\lim _{n \rightarrow \infty} \frac{K_{n}\left(z_{\infty}, z_{\infty} ; \nu\right)}{K_{n}\left(z_{\infty}, z_{\infty} ; \mu^{*}\right)}=1
$$

Then

$$
\lim _{n \rightarrow \infty} \frac{K_{n}\left(z_{n}, w_{n} ; \mu\right)}{K_{n}\left(z_{n}, w_{n} ; \nu\right)}=1
$$

REMARK. It is for use with $x_{n}=x_{\infty}+\frac{a}{n}$ or $x_{\infty}+\frac{a}{\rho_{n} n}$ that we added $x_{n} \rightarrow x_{\infty}$ to the various diagonal kernel results. This "wiggle" in $x_{\infty}$ was introduced by Lubinsky [60], so we dub it the "Lubinsky wiggle."

Given Totik's theorem (Theorem 17.3) and bulk universality for suitable models, one thus gets:

THEOREM 20.3. Under the hypotheses of Theorem 17.3, for a.e. $x_{\infty}$ in I, we have uniformly for $|a|,|b|<A$,

$$
\lim _{n \rightarrow \infty} \frac{K_{n}\left(x_{\infty}+\frac{a}{n}, x_{\infty}+\frac{b}{n}\right)}{K_{n}\left(x_{\infty}, x_{\infty}\right)}=\frac{\sin \left(\pi \rho_{\mathfrak{e}}\left(x_{\infty}\right)(b-a)\right)}{\pi \rho_{\mathfrak{e}}\left(x_{\infty}\right)(b-a)}
$$

Remarks. 1. For $\mathfrak{e}=[-1,1]$, the result and method are from Lubinsky $\underline{\mathbf{6 0}}$.

2. For continuous weights, this is in Simon [87] and Totik [99], and for general weights, in Totik [99].

\section{Derivatives of CD Kernels}

The ideas in this section come from a paper in preparation with Avila and Last [6. Variation of parameters is a standard technique in ODE theory and used as an especially powerful tool in spectral theory by Gilbert-Pearson [38] and in Jacobi matrix spectral theory by Khan-Pearson [49]. It was then developed by Jitomirskaya-Last [4, 45, 46] and Killip-Kiselev-Last [50, from which we take Proposition 21.1. 
Proposition 21.1. For any $x, x_{0}$, we have

$$
p_{n}(x)-p_{n}\left(x_{0}\right)=\left(x-x_{0}\right) \sum_{m=0}^{n-1}\left(p_{n}\left(x_{0}\right) q_{m}\left(x_{0}\right)-p_{m}\left(x_{0}\right) q_{n}\left(x_{0}\right)\right) p_{m}(x)
$$

In particular,

$$
p_{n}^{\prime}\left(x_{0}\right)=\sum_{m=0}^{n-1}\left(p_{n}\left(x_{0}\right) q_{m}\left(x_{0}\right)-p_{m}\left(x_{0}\right) q_{n}\left(x_{0}\right)\right) p_{m}\left(x_{0}\right)
$$

Here $q_{n}$ are the second kind polynomials defined in Section 8 . For (21.1), see 44, 45, 46, 50. This immediately implies:

Corollary 21.2 (Avila-Last-Simon [6]).

$$
\begin{aligned}
\frac{d}{d a} & \left.\frac{1}{n} K_{n}\left(x_{0}+\frac{a}{n}, x_{0}+\frac{a}{n}\right)\right|_{a=0} \\
\quad= & \frac{2}{n^{2}} \sum_{j=0}^{n}\left[p_{j}\left(x_{0}\right)^{2}\left(\sum_{k=0}^{j} p_{k}\left(x_{0}\right) q_{k}\left(x_{0}\right)\right)-q_{j}\left(x_{0}\right) p_{j}\left(x_{0}\right)\left(\sum_{k=0}^{j} p_{k}\left(x_{0}\right)^{2}\right)\right]
\end{aligned}
$$

This formula gives an indication of why (as we see in the next section is important) $\lim \frac{1}{n} K_{n}\left(x_{0}+\frac{a}{n}, x_{0}+\frac{a}{n}\right)$ has a chance to be independent of $a$ if one notes the following fact:

LEMMA 21.3. If $\left\{\alpha_{n}\right\}_{n=1}^{\infty}$ and $\left\{\beta_{n}\right\}_{n=1}^{\infty}$ are sequences so that $\lim \frac{1}{N} \sum_{n=1}^{N} \alpha_{n}=$ $A$ and $\lim \frac{1}{N} \sum_{n=1}^{N} \beta_{n}=B$ exist and $\sup _{N}\left[\frac{1}{N} \sum_{n=1}^{N}\left|\alpha_{n}\right|+\left|\beta_{n}\right|\right]<\infty$, then

$$
\frac{1}{N^{2}} \sum_{j=1}^{N}\left[\left(\alpha_{j} \sum_{k=1}^{j} \beta_{k}\right)-\left(\beta_{j} \sum_{k=1}^{j} \alpha_{k}\right)\right] \rightarrow 0
$$

This is because

$$
\frac{1}{N^{2}} \sum_{j=1}^{N} \alpha_{j} \sum_{k=1}^{j} \beta_{k} \rightarrow \frac{1}{2} A B
$$

Setting $\alpha_{j}=p_{j}\left(x_{0}\right)^{2}$ and $\beta_{j}=p_{j}\left(x_{0}\right) q_{j}\left(x_{0}\right)$, one can hope to use (21.4) to prove the right side of (21.3) goes to zero.

\section{Lubinsky's Second Approach}

Lubinsky revolutionized the study of universality in $6 \mathbf{6 0}$, introducing the approach we described in Section 20. While Totik 99. and Simon 87. used those ideas to extend beyond the case of $\mathfrak{e}=[-1,1]$ treated in $\mathbf{6 0}$, Lubinsky developed a totally different approach 61] to go beyond [60. That approach, as abstracted in Avila-Last-Simon [6], is discussed in this section. Here is an abstract theorem:

THEOREM 22.1. Let $d \mu$ be a measure of compact support on $\mathbb{R}$. Let $x_{0}$ be a Lebesgue point for $\mu$ and suppose that

(i) For any $\varepsilon$, there is a $C_{\varepsilon}$ so that for any $R$, we have an $N(\varepsilon, R)$ so that for $n \geq N(\varepsilon, R)$

$$
\frac{1}{n} K_{n}\left(x_{0}+\frac{z}{n}, x_{0}+\frac{z}{n}\right) \leq C_{\varepsilon} e^{\varepsilon|z|^{2}}
$$

for all $z \in \mathbb{C}$ with $|z|<R$. 
(ii) Uniformly for real a's in compact subsets of $\mathbb{R}$,

$$
\lim _{n \rightarrow \infty} \frac{K_{n}\left(x_{0}+\frac{a}{n}, x_{0}+\frac{a}{n}\right)}{K_{n}\left(x_{0}, x_{0}\right)}=1
$$

Let

$$
\rho_{n}=\frac{w\left(x_{0}\right)}{n} K_{n}\left(x_{0}, x_{0}\right)
$$

Then uniformly for $z, w$ in compact subsets of $\mathbb{C}$,

$$
\lim _{n \rightarrow \infty} \frac{K_{n}\left(x_{0}+\frac{z}{n \rho_{n}}, x_{0}+\frac{w}{n \rho_{n}}\right)}{K_{n}\left(x_{0}, x_{0}\right)}=\frac{\sin (\pi(\bar{z}-w))}{\pi(\bar{z}-w)}
$$

Remarks. 1. If $\rho_{n} \rightarrow \rho_{\mathfrak{e}}\left(x_{0}\right)$, the density of the equilibrium measure, then (22.4) is the same as (19.2). In every case where Theorem 22.1 has been proven to be applicable (see below), $\rho_{n} \rightarrow \rho_{\mathfrak{e}}\left(x_{0}\right)$. But one of the interesting aspects of this is that it might apply in cases where $\rho_{n}$ does not have a limit. For an example with a.c. spectrum but where the density of zeros has multiple limits, see Example 5.8 of 85 .

2. Lubinsky 61] worked in a situation (namely, $x_{0}$ in an interval $I$ with $w\left(x_{0}\right) \geq$ $c>0$ on $I$ ) where (22.1) holds in the stronger form $C e^{D|z|}$ (no square on $|z|$ ) and used arguments that rely on this. Avila-Last-Simon [6] found the result stated here; the methods seem incapable of working with (22.1) for a fixed $\varepsilon$ rather than all $\varepsilon$ (see Remark 1 after Theorem 22.2).

Let us sketch the main ideas in the proof of Theorem 22.1

(1) By (15.1),

$$
\liminf \frac{1}{n} K_{n}\left(x_{0}, x_{0}\right)>0
$$

(2) By the Schwarz inequality (1.14), (22.1), and (22.5), and by the compactness of normal families, we can find subsequences $n(j)$ so

$$
\frac{K_{n(j)}\left(x_{0}+\frac{z}{n \rho_{n}}, x_{0}+\frac{w}{n \rho_{n}}\right)}{K_{n(j)}\left(x_{0}, x_{0}\right)} \rightarrow F(z, w)
$$

and $F$ is analytic in $w$ and anti-analytic in $z$.

(3) Note that by (22.2) and the Schwarz inequality (1.14), we have for $a, b \in \mathbb{R}$,

$$
F(a, a)=1 \quad|F(a, b)| \leq 1
$$

By compactness, if we show any such limiting $F$ is $\sin (\pi(\bar{z}-w)) /(\bar{z}-w)$, we have (22.4). By analyticity, it suffices to prove this for $z=a$ real, and we will give details when $z=0$, that is, we consider

$$
\frac{K_{n(j)}\left(x_{0}, x_{0}+\frac{z}{n \rho_{n}}\right)}{K_{n(j)}\left(x_{0}, x_{0}\right)} \rightarrow f(z)
$$

(22.7) becomes

$$
f(0)=1 \quad|f(x)| \leq 1 \text { for } x \text { real }
$$

(4) By (1.19),

$$
\int\left|K_{n}\left(x_{0}, x_{0}+a\right)\right|^{2} w(a) d a \leq K_{n}\left(x_{0}, x_{0}\right)
$$


which, by using the fact that $x_{0}$ is Lebesgue point, can be used to show

$$
\int_{-\infty}^{\infty}|f(x)|^{2} d x \leq 1
$$

(5) By properties of $K_{n}$ (see Section [6) and Hurwitz's theorem, $f$ has zeros $\left\{x_{j}\right\}_{j=-\infty, j \neq 0}^{\infty}$ only on $\mathbb{R}$, which we label by

$$
\cdots<x_{-1}<0<x_{1}<x_{2}<\cdots
$$

and define $x_{0}=0$. By Theorem 7.2 using (22.2), we have for any $j, k$ that

$$
\left|x_{j}-x_{k}\right| \geq|j-k|-1
$$

(6) Given these facts, the theorem is reduced to

$$
f(0)=1 \quad|f(x)| \leq 1 \text { for } x \text { real }
$$

$$
\int_{-\infty}^{\infty}|f(x)|^{2} d x \leq 1
$$

(3) $f$ is real on $\mathbb{R}$, has only real zeros, and if they are labelled by (22.11), then (22.12) holds.

(4) For any $\varepsilon$, there is a $C_{\varepsilon}$ so

$$
|f(z)| \leq C_{\varepsilon} e^{\varepsilon|z|^{2}}
$$

Then

$$
f(z)=\frac{\sin \pi z}{\pi z}
$$

Remarks. 1. There exist examples ([6] $) e^{-a z^{2}+b z} \sin \pi z / \pi z$ that obey (1)-(3) and (22.15) for some but not all $\varepsilon$.

2. We sketch the proof of this in case one has

$$
|f(z)| \leq C e^{D|z|}
$$

instead of (22.15); see [6] for the general case.

Lemma 22.3. If (1)-(3) hold and (22.17) holds, then for any $\varepsilon>0$, there is $D_{\varepsilon}$ with

$$
|f(z)| \leq D_{\varepsilon} e^{(\pi+\varepsilon)|\operatorname{Im} z|}
$$

Sketch. By the Hadamard product formula [2,

$$
f(z)=e^{D z} \prod_{j \neq 0}\left(1-\frac{z}{x_{j}}\right) e^{z x_{j}}
$$

where $D$ is real since $f$ is real on $\mathbb{R}$. Thus, for $y$ real,

$$
|f(i y)|^{2}=\prod_{j \neq 0}\left(1+\frac{y^{2}}{x_{j}^{2}}\right)
$$

By (22.12), $\left|x_{j}\right| \geq j-1$, so

$$
|f(i y)|^{2} \leq\left(1+\frac{y^{2}}{x_{1}^{2}}\right)\left(1+\frac{y^{2}}{x_{-1}^{2}}\right)\left[\prod_{j=1}^{\infty}\left(1+\frac{y^{2}}{j^{2}}\right)\right]^{2}
$$


which, given Euler's formula for $\sin \pi z / z$, implies (22.18) for $z=i y$. By a Phragmén-Lindelöf argument, (22.18) for $z$ real and for $z$ pure imaginary and (22.17) implies (22.18) for all $z$.

Thus, Theorem 22.2 (under hypothesis (22.17)) is implied by:

LEMma 22.4. If $f$ is an entire function that obeys (22.13), (22.14), and (22.18), then (22.16) holds.

Proof. Let $\hat{f}$ be the Fourier transform of $f$, that is,

$$
\hat{f}(k)=(2 \pi)^{-1 / 2} \int e^{-i k x} f(x) d x
$$

(in $L^{2}$ limit sense). By the Paley-Wiener Theorem [74, (22.18) implies $\hat{f}$ is supported on $[-\pi, \pi]$. By (22.14),

$$
\|\hat{f}\|_{L^{2}}=\left\|(2 \pi)^{-1 / 2} \chi_{[-\pi, \pi]}\right\|_{L^{2}}=1
$$

and, by (22.13) and support property of $\hat{f}$,

$$
\left\langle\hat{f},(2 \pi)^{-1 / 2} \chi_{[-\pi, \pi]}\right\rangle=1
$$

We thus have equality in the Schwarz inequality, so

$$
\hat{f}=(2 \pi)^{-1 / 2} \chi_{[-\pi, \pi]}
$$

which implies (22.16).

This theorem has been applied in two ways:

(a) Lubinsky 61 noted that one can recover Theorem 20.3 from just Totik's result Theorem 17.3 without using the Lubinsky wiggle or Lubinsky's inequality.

(b) Avila-Last-Simon [6] have used this result to prove universality for ergodic Jacobi matrices with a.c. spectrum where $\mathfrak{e}$ can be a positive measure Cantor set.

\section{Zeros: The Freud-Levin-Lubinsky Argument}

In the final section of his book [34, Freud proved bulk universality under fairly strong hypotheses on measures on $[-1,1]$ and noticed that it implied a strong result on local equal spacings of zeros. Without knowing of Freud's work, Simon, in a series of papers (one joint with Last) $[\mathbf{8 2}, \mathbf{8 3}, \mathbf{8 4},[\mathbf{5 7}$, focused on this behavior, called it clock spacing, and proved it in a variety of situations (not using universality or the CD kernel). After Lubinsky's work on universality, Levin 59 rediscovered Freud's argument and Levin-Lubinsky [59] used this to obtain clock behavior in a very general context. Here is an abstract version of their result:

TheOREM 23.1. Let $\mu$ be a measure of compact support on $\mathbb{R}$; let $x_{0} \in \sigma(\mu)$ be such that for each $A$, for some $c_{n}$,

$$
\frac{K_{n-1}\left(x_{0}+\frac{a}{n c_{n}}, x_{0}+\frac{b}{n c_{n}}\right)}{K_{n-1}\left(x_{0}, x_{0}\right)} \rightarrow \frac{\sin (\pi(b-a))}{\pi(b-a)}
$$

uniformly for real $a, b$ with $|a|,|b| \leq A$. Let $x_{j}^{(n)}\left(x_{0}\right)$ denote the zeros of $p_{n}(x ; d \mu)$ labelled so

$$
\cdots<x_{-1}^{(n)}\left(x_{0}\right)<x_{0} \leq x_{0}^{(n)}\left(x_{0}\right)<x_{1}^{(n)}\left(x_{0}\right)<\cdots
$$

Then 
(1)

$$
\limsup n c_{n}\left(x_{0}^{(n)}-x_{0}\right) \leq 1
$$

(ii) For any $J$, for large $n$, there are zeros $x_{j}^{(n)}$ for all $j \in\{-J,-J+1, \ldots, J-1, J\}$. (iii)

$$
\lim _{n \rightarrow \infty}\left(x_{j+1}^{(n)}-x_{j}^{(n)}\right) n c_{n}=1 \quad \text { for each } j
$$

REMARKS. 1. The meaning of $x_{j}^{(n)}$ has changed slightly from Section 6 ,

2. Only $n c_{n}$ enters, so the " $n$ " could be suppressed; we include it because one expects $c_{n}$ as defined to be bounded above and below. Indeed, in all known cases, $c_{n} \rightarrow \rho\left(x_{0}\right)$, the derivative of the density of states. But see Remark 1 after Theorem 22.1 for cases where $c_{n}$ might not have a limit.

3. See [58] for the OPUC case.

Proof. Let $\tilde{x}_{j}^{(n)}\left(x_{0}\right)$ be the zeros of $p_{n}(x) p_{n-1}\left(x_{0}\right)-p_{n}\left(x_{0}\right) p_{n-1}(x)$ labelled as in (23.2) (with $\left.\tilde{x}_{0}^{(n)}\left(x_{0}\right)=x_{0}\right)$. By (23.1), we have $\tilde{x}_{ \pm 1}^{(n)}\left(x_{0}\right) n c_{n} \rightarrow 1$ since $\sin (\pi a) / a$ is nonvanishing on $(-1,1)$ and vanishes at \pm 1 . The same argument shows $K_{n}\left(\tilde{x}_{ \pm 1}^{(n)}, \tilde{x}_{ \pm 1}^{(n)}+b / n c_{n}\right)$ is nonvanishing for $|b|<\frac{1}{2}$, and so there is at most one zero near $\tilde{x}_{ \pm 1}^{(n)}$ on $1 / n c_{n}$ scale. It follows by repeating this argument that

$$
n c_{n} \tilde{x}_{j}^{(n)} \rightarrow j
$$

for all $j$.

Since we have (see Section 6) that

$$
x_{0} \leq x_{0}^{(n)}\left(x_{0}\right) \leq \tilde{x}_{1}^{(n)}\left(x_{0}\right)
$$

by interlacing, which implies (i) and similar interlacing gives (ii). Finally, (23.4) follows from the same argument that led to (23.5).

\section{Adding Point Masses}

We end with a final result involving CD kernels - a formula of Geronimus [37. formula (3.30)]. While he states it only for OPUC, his proof works for any measure on $\mathbb{C}$ with finite moments. Let $\mu$ be such a measure, let $z_{0} \in \mathbb{C}$, and let

$$
\nu=\mu+\lambda \delta_{z_{0}}
$$

for $\lambda$ real and bigger than or equal to $-\mu\left(\left\{z_{0}\right\}\right)$.

Since $X_{n}(z ; d \nu)$ and $X_{n}(z ; d \mu)$ are both monic, their difference is a polynomial of degree $n-1$, so

$$
X_{n}(x ; d \nu)=X_{n}(z ; d \mu)+\sum_{j=0}^{n-1} c_{j} x_{j}(z ; d \mu)
$$

where

$$
\begin{aligned}
c_{j} & =\int \overline{x_{j}(z ; d \mu)}\left[X_{n}(z ; d \nu)-X_{n}(z ; d \mu)\right] d \mu \\
& =\int \overline{x_{j}(z ; d \mu)} X_{n}(z ; d \nu)\left[d \nu-\lambda \delta_{z_{0}}\right] \\
& =-\lambda \overline{x_{j}\left(z_{0} ; d \mu\right)} X_{n}\left(z_{0} ; d \nu\right)
\end{aligned}
$$


where (24.4) follows from $x_{j}(\cdot, d \mu) \perp X_{n}(\cdot, d \mu)$ in $L^{2}(d \mu)$ and (24.5) from $x_{j}(\cdot, d \mu) \perp X_{n}(\cdot, d \nu)$ in $L^{2}(d \nu)$. Thus,

$$
X_{n}(z ; d \nu)=X_{n}(z ; d \mu)-\lambda X_{n}\left(z_{0} ; d \nu\right) K_{n-1}\left(z_{0}, z ; d \mu\right)
$$

Set $z=z_{0}$ and solve for $X_{n}\left(z_{0} ; d \nu\right)$ to get:

Theorem 24.1 (Geronimus [37). Let $\mu, \nu$ be related by (24.1). Then

$$
X_{n}(z ; d \nu)=X_{n}(z ; d \mu)-\frac{\lambda X_{n}\left(z_{0} ; d \mu\right) K_{n-1}\left(z_{0}, z ; d \mu\right)}{1+\lambda K_{n-1}\left(z_{0}, z_{0} ; d \mu\right)}
$$

This formula was rediscovered by Nevai 67 for OPRL, by CachafeiroMarcellán [15, 16, Simon 81] (in a weak form), and Wong [105, 106] for OPUC. For general measures on $\mathbb{C}$, the formula is from Cachafeiro-Marcellán [17, 18. In particular, in the context of OPUC, Wong [106] noted that one can use the CD formula to obtain:

Theorem 24.2 (Wong [105, 106 ). Let $d \mu$ be a probability measure on $\partial \mathbb{D}$ and let $d \tilde{\nu}$ be given by

$$
d \tilde{\nu}=\frac{d \mu+\lambda \delta_{z_{0}}}{1+\lambda}
$$

for $z_{0} \in \partial \mathbb{D}$ and $\lambda \geq-\mu\left(\left\{z_{0}\right\}\right)$. Then

$$
\alpha_{n}(d \tilde{\nu})=\alpha_{n}(d \mu)+\frac{\left(1-\left|\alpha_{n}(d \mu)\right|^{2}\right)^{1 / 2}}{\lambda^{-1}+K_{n}\left(z_{0}, z_{0} ; d \mu\right)} \overline{\varphi_{n+1}\left(z_{0}\right)} \varphi_{n}^{*}\left(z_{0}\right)
$$

Proof. Let

$$
Q_{n}=\lambda^{-1}+K_{n}\left(z_{0}, z ; d \mu\right)
$$

Since

$$
\Phi_{n+1}(z ; d \tilde{\nu})=\Phi_{n+1}(z ; d \nu)
$$

and

(24.7) becomes

$$
\alpha_{n}(d \tilde{\nu})=\overline{-\Phi_{n+1}(0 ; d \tilde{\nu})}
$$

$$
\alpha_{n}(d \tilde{\nu})-\alpha_{n}(d \mu)=Q_{n}^{-1} \overline{\Phi_{n+1}\left(z_{0}\right)} \overline{K_{n}\left(z_{0}, 0 ; d \mu\right)}
$$

By the CD formula in the form (3.23),

$$
\begin{aligned}
\overline{K_{n}\left(z_{0}, 0\right)} & =\varphi_{n}^{*}\left(z_{0}\right) \overline{\varphi_{n}^{*}(0)} \\
& =\frac{\varphi_{n}^{*}\left(z_{0}\right)}{\left\|\Phi_{n}\right\|}
\end{aligned}
$$

since $\Phi_{n}^{*}(0)=1$ and $\left\|\Phi_{n}^{*}\right\|=\left\|\Phi_{n}\right\|$. (24.9) then follows from $\left\|\Phi_{n+1}\right\| /\left\|\Phi_{n}\right\|=$ $\left(1-\left|\alpha_{n}\right|^{2}\right)^{1 / 2}$.

To see a typical application:

Corollary 24.3. Let $z_{0}$ be an isolated pure point of a measure $d \mu$ on $\partial \mathbb{D}$. Let $d \tilde{\nu}$ be given by (24.8) where $\lambda>-\mu\left(\left\{z_{0}\right\}\right)$ (so $z_{0}$ is also a pure point of $\left.d \tilde{\nu}\right)$. Then for some $D, C>0$,

$$
\left|\alpha_{n}(d \tilde{\nu})-\alpha_{n}(d \mu)\right| \leq D e^{-C n}
$$

Proof. By Theorem 10.14.2 of 81,

$$
\left|\varphi_{n}\left(z_{0} ; d \mu\right)\right| \leq D_{1} e^{-\frac{1}{2} C n}
$$

This plus (24.9) implies (24.16). 
This is not only true for OPUC but also for OPRL:

Corollary 24.4. Let $z_{0}$ be an isolated pure point of a measure of compact support $d \mu$ on $\mathbb{R}$. Let dĩ be given by (24.8) where $\lambda>-\mu\left(\left\{z_{0}\right\}\right)$ so $z$ is also a pure point of $d \tilde{\nu}$. Then for some $D, C>0$,

$$
\begin{aligned}
& \left|\frac{\kappa_{n}(d \nu)}{\kappa_{n}(d \mu)}-(1+\lambda)^{1 / 2}\right| \leq D e^{-C n} \\
& \left\|p_{n}(\cdot, d \nu)-(1+\lambda)^{1 / 2} p_{n}(\cdot, d \mu)\right\|_{L^{2}(d \nu)} \leq D e^{-C n} \\
& \left|a_{n}(d \nu)-a_{n}(d \mu)\right| \leq D e^{-C n} \\
& \left|b_{n}(d \nu)-b_{n}(d \mu)\right| \leq D e^{-C n}
\end{aligned}
$$

SkETCH. Isolated points in the spectrum of Jacobi matrices obey

$$
\left|p_{n}\left(z_{0}\right)\right| \leq D_{1} e^{-C_{1} n}
$$

for suitable $C_{1}, D_{1}$ (see [1, 25]).

(24.7) can be rewritten for OPRL

$$
\kappa_{n}(d \mu) P_{n}(x ; d \nu)=p_{n}(x ; d \mu)-\lambda p_{n}\left(x_{0} ; d \mu\right) \frac{K_{n-1}\left(x_{0}, x ; d \mu\right)}{1+\lambda K_{n}\left(x_{0}, x_{0} ; d \mu\right)}
$$

Since

and

$$
\left\|K_{n-1}\left(x_{0}, x ; d \mu\right)\right\|_{L^{2}(d \mu)}^{2}=K_{n-1}\left(x_{0}, x_{0} ; d \mu\right)
$$

$$
\int\left|K_{n-1}\left(x_{0}, x_{0} ; d \mu\right)\right|^{2} d \delta_{x_{0}}=K_{n-1}\left(x_{0}, x_{0} ; d \mu\right)^{2}
$$

and $K_{n-1}\left(x_{0}, x_{0}\right)$ is bounded (by (24.22)), we see that $\left\|K_{n-1}\left(x_{0} ; \cdot ; d \mu\right)\right\|_{L^{2}(d \nu)}$ is bounded. Thus, by (24.22) and (24.23),

$$
\kappa_{n}(d \mu) \kappa_{n}(d \nu)^{-1}=(1+\lambda)^{-1 / 2}+O\left(e^{-C_{1} n}\right)
$$

which leads to (24.18).

This in turn leads to (ii), and that to (iii) via (24.22), and, for example,

$$
\begin{aligned}
& a_{n}(d \mu)=\int x p_{n}(x ; d \mu) p_{n-1}(x ; d \mu) d \mu \\
& a_{n}(d \nu)=\int x p_{n}(x ; d \nu) p_{n-1}(x ; d \nu) d \nu
\end{aligned}
$$

This shows what happens if the weight of an isolated eigenvalue changes. What happens if an isolated eigenvalue is totally removed is much more subtle - sometimes it is exponentially small, sometimes not. This is studied by Wong [107.

\section{References}

[1] S. Agmon, Lectures on Exponential Decay of Solutions of Second-Order Elliptic Equations: Bounds on Eigenfunctions of $N$-body Schrödinger Operators, Mathematical Notes, 29, Princeton Univ. Press, Princeton, NJ; Univ. of Tokyo Press, Tokyo, 1982.

[2] L. V. Ahlfors, Complex Analysis. An Introduction to the Theory of Analytic Functions of One Complex Variable, McGraw-Hill, New York, 1978.

[3] A. Ambroladze, On exceptional sets of asymptotic relations for general orthogonal polynomials, J. Approx. Theory 82 (1995), 257-273. 
[4] A. I. Aptekarev, Asymptotic properties of polynomials orthogonal on a system of contours, and periodic motions of Toda chains, Math. USSR Sb. 53 (1986), 233-260; Russian original in Mat. Sb. (N.S.) 125(167) (1984), 231-258.

[5] F. V. Atkinson, Discrete and Continuous Boundary Problems, Academic Press, New York, 1964.

[6] A. Avila, Y. Last, and B. Simon, Bulk universality and clock spacing of zeros for ergodic Jacobi matrices with a.c. spectrum, in preparation.

[7] J. Avron and B. Simon, Almost periodic Schrödinger operators, II. The integrated density of states, Duke Math. J. 50 (1983), 369-391.

[8] D. Barrios Rolanía and G. López Lagomasino, Ratio asymptotics for polynomials orthogonal on arcs of the unit circle, Constr. Approx. 15 (1999), 1-31.

[9] M. Bello Hernández and G. López Lagomasino, Ratio and relative asymptotics of polynomials orthogonal on an arc of the unit circle, J. Approx. Theory 92 (1998), 216-244.

[10] A. Ben Artzi and T. Shalom, On inversion of block Toeplitz matrices, Integral Equations and Operator Theory 8 (1985), 751-779.

[11] C. Berg, Fibonacci numbers and orthogonal polynomials, to appear in J. Comput. Appl. Math.

[12] A. B. Bogatyrëv, On the efficient computation of Chebyshev polynomials for several intervals, Sb. Math. 190 (1999), 1571-1605; Russian original in Mat. Sb. 190 (1999), no. 11, $15-50$.

[13] A. Borodin, Biorthogonal ensembles, Nuclear Phys. B 536 (1999), 704-732.

[14] J. Breuer, Y. Last, and B. Simon, in preparation.

[15] A. Cachafeiro and F. Marcellán, Orthogonal polynomials and jump modifications, in "Orthogonal Polynomials and Their Applications," (Segovia, 1986), pp. 236-240, Lecture Notes in Math., 1329, Springer, Berlin 1988.

[16] A. Cachafeiro and F. Marcellán, Asymptotics for the ratio of the leading coefficients of orthogonal polynomials associated with a jump modification, in "Approximation and Optimization," (Havana, 1987), pp. 111-117, Lecture Notes in Math., 1354, Springer, Berlin, 1988.

[17] A. Cachafeiro and F. Marcellán, Perturbations in Toeplitz matrices, in "Orthogonal Polynomials and Their Applications," (Laredo, 1987), pp. 139-146, Lecture Notes in Pure and Applied Math., 117, Marcel Dekker, New York, 1989.

[18] A. Cachafeiro and F. Marcellán, Perturbations in Toeplitz matrices: Asymptotic properties, J. Math. Anal. Appl. 156 (1991) 44-51.

[19] M. J. Cantero, L. Moral, and L. Velázquez, Measures and para-orthogonal polynomials on the unit circle, East J. Approx. 8 (2002), 447-464.

[20] R. Carmona and J. Lacroix, Spectral Theory of Random Schrödinger Operators. Probability and Its Applications, Birkhäuser, Boston, 1990.

[21] M. P. Chebyshev, Sur les valeurs limites des intégrales, J. Math. Pures Appl. 19 (1874), 157-160.

[22] T. S. Chihara, An Introduction to Orthogonal Polynomials, Mathematics and Its Applications, 13, Gordon and Breach, New York-London-Paris, 1978.

[23] E. B. Christoffel, Über die Gaussische Quadratur und eine Verallgemeinerung derselben, J. Reine Angew. Math. 55 (1858), 61-82.

[24] A. R. Collar, On the reciprocation of certain matrices, Proc. Roy. Soc. Edinburgh 59 (1939), 195-206.

[25] J. M. Combes and L. Thomas, Asymptotic behaviour of eigenfunctions for multiparticle Schrödinger operators, Comm. Math. Phys. 34 (1973), 251-270.

[26] D. Damanik, R. Killip, and B. Simon, Perturbations of orthogonal polynomials with periodic recursion coefficients, preprint.

[27] D. Damanik, A. Pushnitski, and B. Simon, The analytic theory of matrix orthogonal polynomials, Surveys in Approximation Theory 4 (2008), 1-85.

[28] G. Darboux, Mémoire sur l'approximation des fonctions de très-grands nombres, et sur une classe étendue de développements en série, Liouville J. (3) 4 (1878), 5-56; 377-416.

[29] S. Denisov and B. Simon, Zeros of orthogonal polynomials on the real line, J. Approx. Theory 121 (2003), 357-364.

[30] J. L. Doob, Measure Theory, Graduate Texts in Math., 143, Springer-Verlag, New York, 1994. 
[31] D. E. Edmunds and W. D. Evans, Spectral Theory and Differential Operators, Clarendon Press, Oxford, 1989.

[32] P. Erdős and P. Turán, On interpolation. III. Interpolatory theory of polynomials, Ann. of Math. (2) 41 (1940), 510-553.

[33] E. Findley, Universality for locally Szegő measures, to appear in J. Approx. Theory.

[34] G. Freud, Orthogonal Polynomials, Pergamon Press, Oxford-New York, 1971.

[35] P. A. Fuhrmann, On a partial realization problem and the recursive inversion of Hankel and Toeplitz matrices, Contemp. Math. 47 (1985), 149-161.

[36] J. S. Geronimo and W. Van Assche, Orthogonal polynomials on several intervals via a polynomial mapping, Trans. Amer. Math. Soc. 308 (1988), 559-581.

[37] Ya. L. Geronimus, Orthogonal Polynomials: Estimates, Asymptotic Formulas, and Series of Polynomials Orthogonal on the Unit Circle and on an Interval, Consultants Bureau, New York, 1961.

[38] D. J. Gilbert and D. B. Pearson, On subordinacy and analysis of the spectrum of onedimensional Schrödinger operators, J. Math. Anal. Appl. 128 (1987), 30-56.

[39] I. C. Gohberg and N. Ya. Krupnik, A formula for the inversion of finite Toeplitz matrices, Mat. Issled 7(2) (1972), 272-283. [Russian]

[40] I. C. Gohberg and A. A. Semencul, On the inversion of finite Toeplitz matrices and their continuous analogs, Mat. Issled 7(2) (1972) 201-223. [Russian]

[41] L. Golinskii, Quadrature formula and zeros of para-orthogonal polynomials on the unit circle, Acta Math. Hungar. 96 (2002), 169-186.

[42] L. L. Helms, Introduction to Potential Theory, Pure and Applied Math., 22, WileyInterscience, New York, 1969.

[43] H. Hochstadt, On the theory of Hill's matrices and related inverse spectral problems, Linear Algebra and Appl. 11 (1975), 41-52.

[44] S. Jitomirskaya and Y. Last, Dimensional Hausdorff properties of singular continuous spectra, Phys. Rev. Lett. 76 (1996), 1765-1769.

[45] S. Jitomirskaya and Y. Last, Power-law subordinacy and singular spectra, I. Half-line operators, Acta Math. 183 (1999), 171-189.

[46] S. Jitomirskaya and Y. Last, Power law subordinacy and singular spectra, II. Line operators, Comm. Math. Phys. 211 (2000), 643-658.

[47] K. Johansson, Random growth and random matrices, European Congress of Mathematics, Vol. I (Barcelona, 2000), pp. 445-456, Progr. Math., 201, Birkhäuser, Basel, 2001.

[48] T. Kailath, A. Vieira, and M. Morf, Inverses of Toeplitz, innovations and orthogonal polynomials, SIAM Review 20 (1978), 106-119.

[49] S. Khan and D. B. Pearson, Subordinacy and spectral theory for infinite matrices, Helv. Phys. Acta 65 (1992), 505-527.

[50] R. Killip, A. Kiselev, and Y. Last, Dynamical upper bounds on wavepacket spreading, Am. J. Math. 125 (2003), 1165-1198.

[51] A. B. Kuijlaars and M. Vanlessen, Universality for eigenvalue correlations from the modified Jacobi unitary ensemble, Int. Math. Res. Not. 30 (2002), 1575-1600.

[52] N. S. Landkof, Foundations of Modern Potential Theory, Springer-Verlag, Berlin-New York, 1972.

[53] Y. Last, On the measure of gaps and spectra for discrete 1D Schrödinger operators, Comm. Math. Phys. 149 (1992), 347-360.

[54] Y. Last, Spectral theory of Sturm-Liouville operators on infinite intervals: a review of recent developments, in "Sturm-Liouville Theory," pp. 99-120, Birkhäuser, Basel, 2005.

[55] Y. Last and B. Simon, Eigenfunctions, transfer matrices, and absolutely continuous spectrum of one-dimensional Schrödinger operators, Invent. Math. 135 (1999), 329-367.

[56] Y. Last and B. Simon, The essential spectrum of Schrödinger, Jacobi, and CMV operators, J. Anal. Math. 98 (2006), 183-220.

[57] Y. Last and B. Simon, Fine structure of the zeros of orthogonal polynomials, IV. A priori bounds and clock behavior, Comm. Pure Appl. Math. 61 (2008) 486-538.

[58] E. Levin and D. S. Lubinsky, Universality limits involving orthogonal polynomials on the unit circle, Computational Methods and Function Theory 7 (2007), 543-561.

[59] E. Levin and D. S. Lubinsky, Applications of universality limits to zeros and reproducing kernels of orthogonal polynomials, J. Approx. Theory 150 (2008), 69-95. 
[60] D. S. Lubinksy, A new approach to universality limits involving orthogonal polynomials, to appear in Ann. of Math.

[61] D. S. Lubinsky, Universality limits in the bulk for arbitrary measures on compact sets, to appear in J. Anal. Math.

[62] D. S. Lubinsky, A new approach to universality limits at the edge of the spectrum, to appear in Contemp. Math.

[63] A. A. Markov, Démonstration de certaines inégalités de M. Tchébychef, Math. Ann. 24 (1884), 172-180.

[64] A. Máté and P. Nevai, Bernstein's inequality in $L^{p}$ for $0<p<1$ and $(C, 1)$ bounds for orthogonal polynomials, Ann. of Math. (2) 111 (1980), 145-154.

[65] A. Máté, P. Nevai, and V. Totik, Extensions of Szegő's theory of orthogonal polynomials. III, Constr. Approx. 3 (1987), 73-96.

[66] A. Máté, P. Nevai, and V. Totik, Szegö's extremum problem on the unit circle, Ann. of Math. 134 (1991), 433-453.

[67] P. Nevai, Orthogonal polynomials, Mem. Amer. Math. Soc. 18 (1979), no. 213, 185 pp.

[68] P. Nevai, Géza Freud, orthogonal polynomials and Christoffel functions. A case study, J. Approx. Theory 48 (1986), $167 \mathrm{pp.}$

[69] P. Nevai, V. Totik, and J. Zhang, Orthogonal polynomials: their growth relative to their sums, J. Approx. Theory 67 (1991), 215-234.

[70] F. Peherstorfer, Orthogonal and extremal polynomials on several intervals, in "Proc. Seventh Spanish Symposium on Orthogonal Polynomials and Applications (VII SPOA)" (Granada, 1991), J. Comput. Appl. Math. 48 (1993), 187-205.

[71] F. Peherstorfer, Deformation of minimal polynomials and approximation of several intervals by an inverse polynomial mapping, J. Approx. Theory 111 (2001), 180-195.

[72] F. Peherstorfer, Inverse images of polynomial mappings and polynomials orthogonal on them, in "Proc. Sixth International Symposium on Orthogonal Polynomials, Special Functions and their Applications" (Rome, 2001), J. Comput. Appl. Math. 153 (2003), 371-385.

[73] T. Ransford, Potential Theory in the Complex Plane, Press Syndicate of the University of Cambridge, New York, 1995.

[74] M. Reed and B. Simon, Methods of Modern Mathematical Physics, II. Fourier Analysis, Self-Adjointness, Academic Press, New York, 1975.

[75] C. Remling, The absolutely continuous spectrum of Jacobi matrices, preprint.

[76] W. Rudin, Real and Complex Analysis, 3rd edition, McGraw-Hill, New York, 1987.

[77] B. Simon, The classical moment problem as a self-adjoint finite difference operator, Adv. in Math. 137 (1998), 82-203.

[78] B. Simon, Ratio asymptotics and weak asymptotic measures for orthogonal polynomials on the real line, J. Approx. Theory 126 (2004), 198-217.

[79] B. Simon, OPUC on one foot, Bull. Amer. Math. Soc. 42 (2005), 431-460.

[80] B. Simon, Orthogonal Polynomials on the Unit Circle, Part 1: Classical Theory, AMS Colloquium Series, 54.1, American Mathematical Society, Providence, RI, 2005.

[81] B. Simon, Orthogonal Polynomials on the Unit Circle, Part 2: Spectral Theory, AMS Colloquium Series, 54.2, American Mathematical Society, Providence, RI, 2005.

[82] B. Simon, Fine structure of the zeros of orthogonal polynomials, I. A tale of two pictures, Electronic Transactions on Numerical Analysis 25 (2006), 328-268.

[83] B. Simon, Fine structure of the zeros of orthogonal polynomials, II. OPUC with competing exponential decay, J. Approx. Theory 135 (2005), 125-139.

[84] B. Simon, Fine structure of the zeros of orthogonal polynomials, III. Periodic recursion coefficients, Comm. Pure Appl. Math. 59 (2006) 1042-1062.

[85] B. Simon, Equilibrium measures and capacities in spectral theory, Inverse Problems and Imaging 1 (2007), 713-772.

[86] B. Simon, Rank one perturbations and the zeros of paraorthogonal polynomials on the unit circle, J. Math. Anal. Appl. 329 (2007), 376-382.

[87] B. Simon, Two extensions of Lubinsky's universality theorem, to appear in J. Anal. Math.

[88] B. Simon, Weak convergence of CD kernels and applications, to appear in Duke Math. J.

[89] B. Simon, Szegö's Theorem and Its Descendants: Spectral Theory for $L^{2}$ Perturbations of Orthogonal Polynomials, in preparation; to be published by Princeton University Press. 
[90] M. Sodin and P. Yuditskii, Almost periodic Jacobi matrices with homogeneous spectrum, infinite-dimensional Jacobi inversion, and Hardy spaces of character-automorphic functions, J. Geom. Anal. 7 (1997), 387-435.

[91] H. Stahl and V. Totik, General Orthogonal Polynomials, in "Encyclopedia of Mathematics and its Applications," 43, Cambridge University Press, Cambridge, 1992.

[92] T. Stieltjes, Quelques recherches sur la théorie des quadratures dites mécaniques, Ann. Sci. École Norm. Sup. (3) 1 (1884), 409-426.

[93] G. Szegő, Orthogonal Polynomials, Amer. Math. Soc. Colloq. Publ., 23, American Mathematical Society, Providence, RI, 1939; 3rd edition, 1967.

[94] R. Szwarc, A counterexample to subexponential growth of orthogonal polynomials, Constr. Approx. 11 (1995), 381-389.

[95] M. Toda, Theory of Nonlinear Lattices, 2nd edition, Springer Series in Solid-State Sciences, 20, Springer, Berlin, 1989.

[96] V. Totik, Asymptotics for Christoffel functions for general measures on the real line, J. Anal. Math. 81 (2000), 283-303.

[97] V. Totik, Polynomial inverse images and polynomial inequalities, Acta Math. 187 (2001), 139-160.

[98] V. Totik, Christoffel functions on curves and domains, in preparation.

[99] V. Totik, Universality and fine zero spacing on general sets, in preparation.

[100] W. F. Trench, An algorithm for the inversion of finite Toeplitz matrices, J. Soc. Industr. Appl. Math. 12 (1964), 515-522.

[101] W. F. Trench, An algorithm for the inversion of finite Hankel matrices, J. Soc. Industr. Appl. Math 13 (1965), 1102-1107.

[102] M. Tsuji, Potential Theory in Modern Function Theory, reprinting of the 1959 original, Chelsea, New York, 1975.

[103] J. L. Ullman, On the regular behaviour of orthogonal polynomials, Proc. London Math. Soc. (3) 24 (1972), 119-148.

[104] P. van Moerbeke, The spectrum of Jacobi matrices, Invent. Math. 37 (1976), 45-81.

[105] M.-W. L. Wong, First and second kind paraorthogonal polynomials and their zeros, J. Approx. Theory 146 (2007), 282-293.

[106] M.-W. L. Wong, A formula for inserting point masses, to appear in Proc. OPSFA (Marseille, $2007)$.

[107] M.-W. L. Wong, in preparation.

[108] J. Zhang, Relative growth of linear iterations and orthogonal polynomials on several intervals, Linear Algebra Appl. 186 (1993), 97-115.

Mathematics 253-37, California Institute of Technology, Pasadena, CA 91125, U.S.A.

E-mail address: bsimon@caltech.edu

$U R L:$ http://www.math.caltech.edu/people/simon.html 